\title{
Supporting Information: Pseudo-Improper-Dihedral Model for Intrinsically Disordered Proteins
}

\author{
Łukasz Mioduszewski ${ }^{1}$, Bartosz Różycki ${ }^{1}$, Marek Cieplak ${ }^{1}$ \\ ${ }^{1}$ Institute of Physics, Polish Academy of Sciences, Al. Lotników 32/46, 02-668 \\ Warsaw, Poland
}

\section{Contents}

1 Cosine function approximation $\quad 1$

2 Distance and PID angle distributions 2

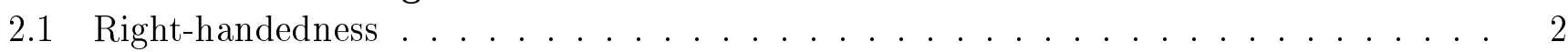

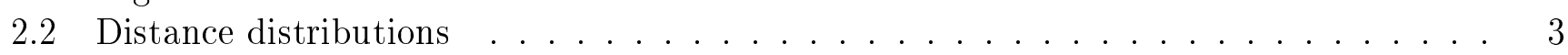

2.3 Backbone-sidechain contacts . . . . . . . . . . . . . . . . . . . . . 4

2.4 Statistics without dividing into bb, bs and ss types ............. 5

3 The parameterization $\quad 6$

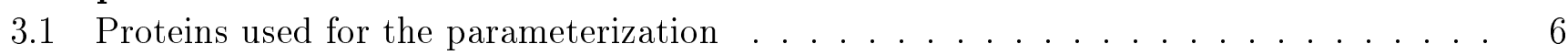

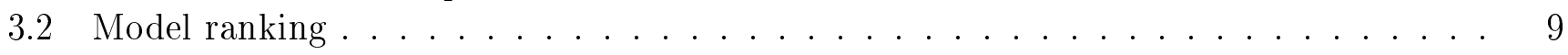

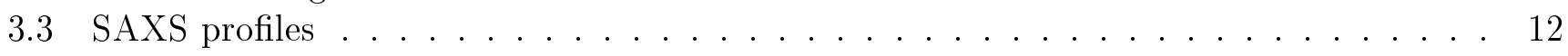

4 Structured proteins $\quad 12$

\section{Cosine function approximation}

We replaced the cosine function by its algebraic approximation to speed the computations up. The approximation is [1]:

$0.5 \cdot \cos v+0.5 \approx \frac{(v / \pi)^{2}-2 \cdot|v / \pi|+1}{2 \cdot(v / \pi)^{2}-2 \cdot|v / \pi|+1}$

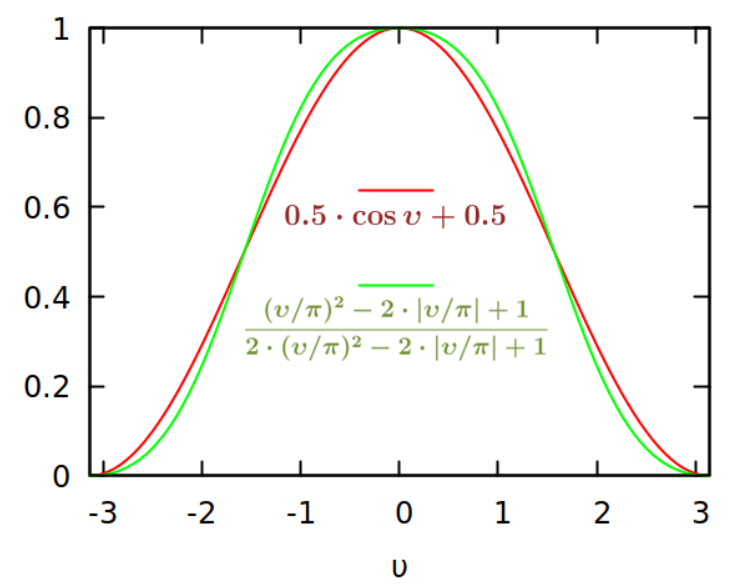

Figure S1: Comparison of the cosine function and its algebraic approximation. 


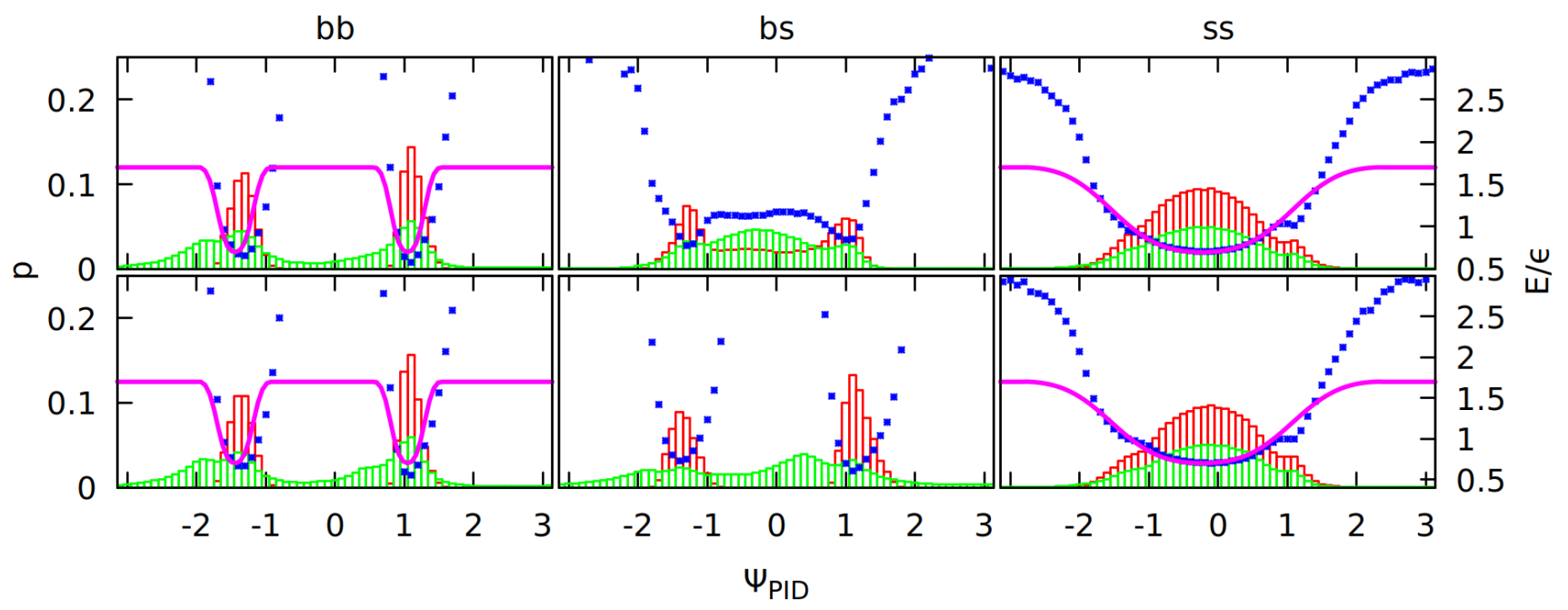

Figure S2: Distributions of the PID angles (in radians) in the contacts from the PDB survey that include a given type (green histograms). Local $i, i+3$ and $i, i+4$ contacts are excluded. Each contact has two angles. Distribution of the first is on the top panels, of the second on the bottom panels. Subdistributions made from contacts that obey the directional criteria defined in [2] are shown as red histograms. The potential resulting from Boltzmann Inversion procedure (blue dots, unit of energy $\epsilon \approx 1.5 \mathrm{kcal} / \mathrm{mol}$ ) was fitted to an analytical function (purple line).

\section{$2.1 \quad$ Right-handedness}

Distributions of PID angles are different for the first and second angle for $i, i+3$ and $i, i+4$ contacts due to the right-handedness of most of $\alpha$ helices (and in our statistics the first PID angle is for the residue earlier in the sequence). This is visible in Fig. S3. Distributions for $i, i+5$ and more nonlocal contacts are mostly symmetric for the operation of exchanging the first and the second PID angles in a contact, with the exception of backbone-sidechain cases (see the subsection about backbone-sidechain contacts).

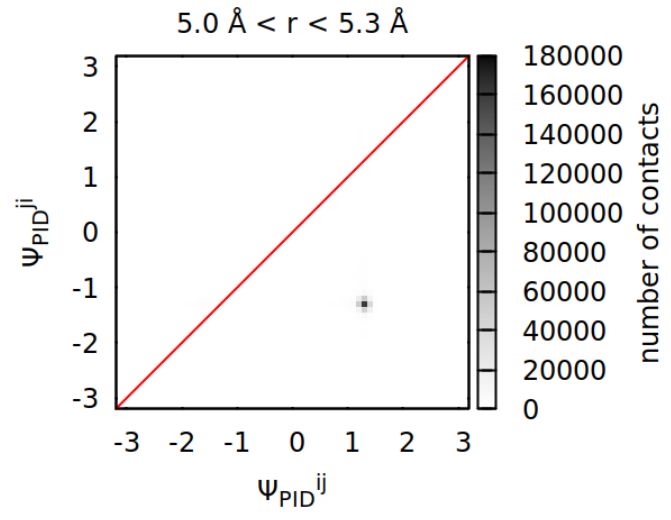

$i, i+3$ contacts

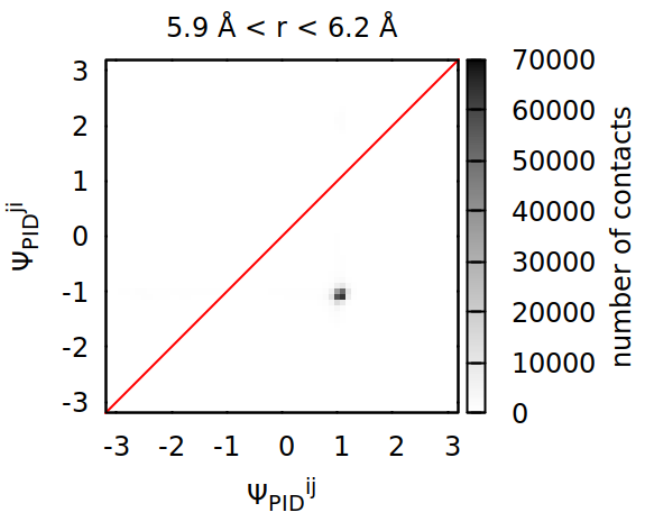

$i, i+4$ contacts

Figure S3: Two-dimensional distribution of backbone-backbone contacts, where the first PID angle in a contact is on one axis and the second PID angle (counting from the $\mathrm{N}$ to $\mathrm{C}$ terminal) is on the other axis. The assymetry probably comes from the right-handedness of an $\alpha$-helix. The distribution is taken for $\mathrm{C}_{\alpha}-\mathrm{C}_{\alpha}$ distances $r$ in a $0.3 \AA$ window to avoid noise from other distances. Note that for those distances the distribution is extremely narrow (each dot has size $0.1 \mathrm{rad}$ x $0.1 \mathrm{rad}$ ). 


\subsection{Distance distributions}

\begin{tabular}{|l|l|l|l|l|l|l|l|l|l|l|l|l|l|l|l|l|l|l|}
\hline$r_{\text {min }}^{\text {ss }}$ & Gln & Cys & Ala & Ser & Val & Thr & Ile & Leu & Asn & Asp & Lys & Glu & Met & His & Phe & Arg & Tyr & Trp \\
\hline Gln & 8.63 & & & & & & & & & & & & & & & & & \\
\hline Cys & 7.72 & 7.56 & & & & & & & & & & & & & & & & \\
\hline Ala & 7.39 & 6.97 & 6.42 & & & & & & & & & & & & & & & \\
\hline Ser & 7.64 & 6.97 & 6.53 & 6.65 & & & & & & & & & & & & & & \\
\hline Val & 7.81 & 7.56 & 7.06 & 7.17 & 7.65 & & & & & & & & & & & & & \\
\hline Thr & 7.77 & 7.40 & 6.94 & 6.97 & 7.54 & 7.30 & & & & & & & & & & & & \\
\hline Ile & 8.24 & 7.95 & 7.45 & 7.52 & 8.06 & 7.93 & 8.53 & & & & & & & & & & & \\
\hline Leu & 8.44 & 8.07 & 7.65 & 7.68 & 8.29 & 8.12 & 8.77 & 8.93 & & & & & & & & & & \\
\hline Asn & 8.19 & 7.49 & 7.02 & 7.18 & 7.54 & 7.46 & 7.96 & 8.14 & 7.74 & & & & & & & & & \\
\hline Asp & 8.15 & 7.18 & 6.73 & 6.99 & 7.22 & 7.19 & 7.65 & 7.86 & 7.50 & & & & & & & & & \\
\hline Lys & 8.69 & 7.83 & 7.26 & 7.73 & 7.69 & 7.79 & 8.16 & 8.39 & 8.11 & 8.59 & & & & & & & & \\
\hline Glu & 8.41 & 7.45 & 7.04 & 7.41 & 7.50 & 7.51 & 7.97 & 8.20 & 8.00 & & 8.90 & & & & & & & \\
\hline Met & 8.84 & 8.29 & 7.91 & 7.94 & 8.48 & 8.33 & 8.95 & 9.14 & 8.49 & 8.15 & 8.80 & 8.61 & 9.29 & & & & & \\
\hline His & 8.64 & 8.17 & 7.50 & 7.88 & 7.92 & 7.98 & 8.37 & 8.57 & 8.36 & 8.50 & 8.58 & 8.84 & 8.93 & 8.83 & & & & \\
\hline Phe & 8.95 & 8.50 & 8.17 & 8.24 & 8.69 & 8.58 & 9.11 & 9.34 & 8.65 & 8.51 & 8.79 & 8.75 & 9.55 & 8.98 & 9.73 & & & \\
\hline Arg & 9.26 & 8.24 & 7.99 & 8.27 & 8.31 & 8.50 & 8.76 & 8.98 & 8.87 & 9.12 & & 9.52 & 9.27 & 9.23 & 9.26 & & & \\
\hline Tyr & 9.27 & 8.26 & 8.02 & 8.36 & 8.39 & 8.58 & 8.78 & 9.02 & 8.96 & 9.35 & 9.04 & 9.48 & 9.28 & 9.38 & 9.56 & 9.51 & 9.34 & \\
\hline Trp & 9.58 & 8.95 & 8.65 & 8.75 & 9.22 & 9.14 & 9.57 & 9.79 & 9.11 & 9.10 & 9.21 & 9.48 & 10.02 & 9.66 & 10.17 & 9.82 & 10.08 & 10.85 \\
\hline
\end{tabular}

Table S1: Average distances, in $\AA$, for the ss contacts as derived from the CATH database, first defined in [2]. Empty boxes indicate same-charged residues that cannot form ss contacts (as well as GLY and PRO). $r_{\text {min }}^{b b+}=5.6 \AA, r_{\text {min }}^{b b-}=6.2 \AA$.

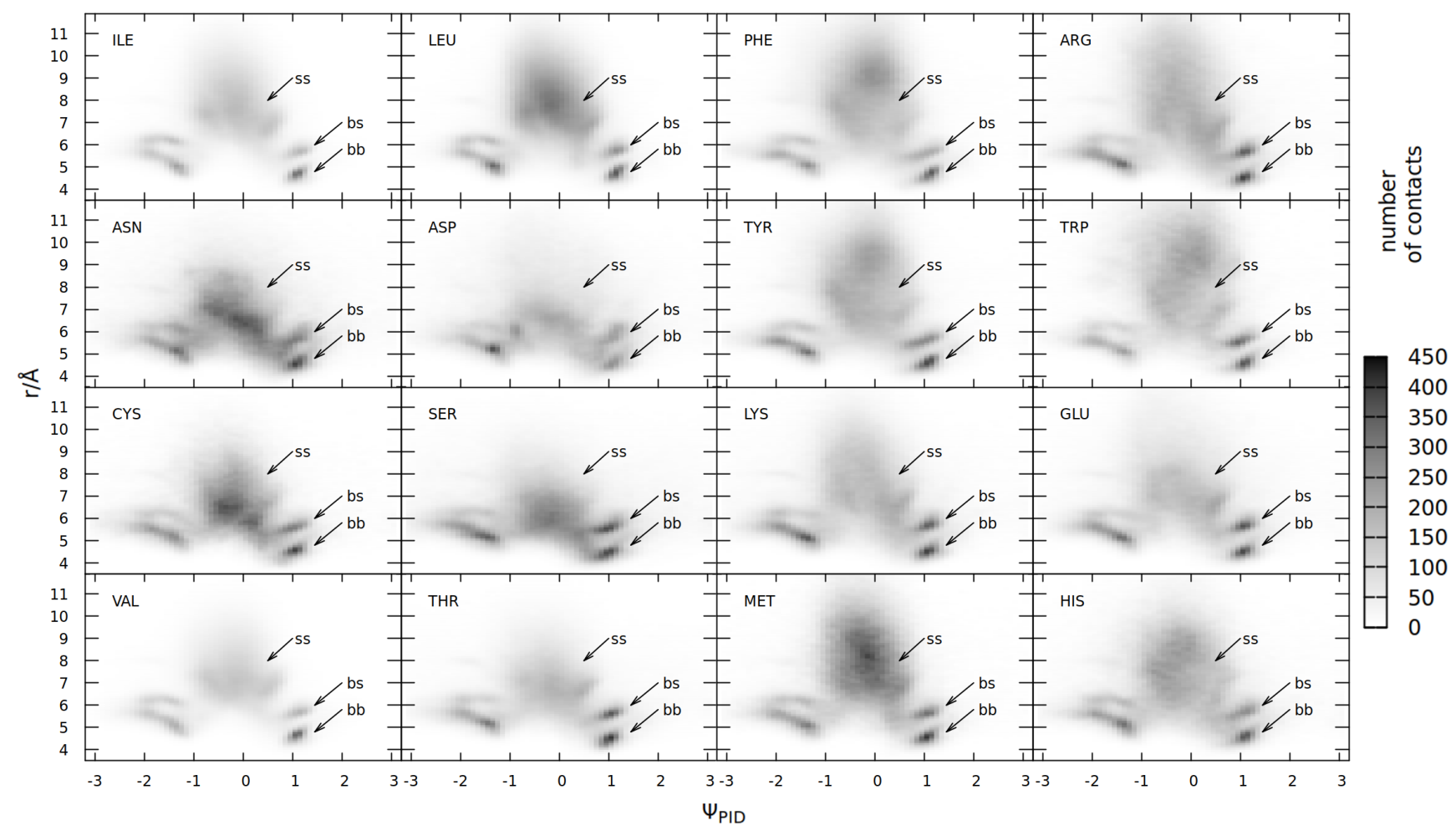

Figure S4: Two-dimensional distributions of contacts, where PID angle (in radians) for a given amino acid is on one axis and $\mathrm{C}_{\alpha}-\mathrm{C}_{\alpha}$ distance is on the other axis. $i, i+3$ and $i, i+4$ contacts are excluded. 


\subsection{Backbone-sidechain contacts}

Backbone-sidechain contacts have very broad distributions of PID angles and $\mathrm{C}_{\alpha}-\mathrm{C}_{\alpha}$ distances. We tried to distinguish the case where the first residue is the backbone and the second is the sidechain (bs) from the opposite case $(\mathrm{sb})$ in the contact distributions that can be of more than one type. The main difference occurs for $\mathrm{C}_{\alpha}-\mathrm{C}_{\alpha}$ distance $5.3 \AA<r<5.6 \AA$, where a peak seems to be associated with a given type of contact (Fig. S5). However, a closer analysis of structures in VMD revealed that this peak corresponds to backbone-backbone contacts, and the sidechain is just a steric hindrance that makes only one combination of PID angles possible. This was confirmed by plotting distributions of contacts belonging to only one overlap type: distribution containing only bb contacts had both peaks (left part of Fig. S6). Most of the bs contacts are also of the bb type (see Table 1 in the main article), so we decided to indicate those mixed bs contacts by arrows in Fig. S4 and in Fig. 3 in the main article.

The only bs (containing also sb) distribution (right part of Fig. S6) was very broad and showed a combination of two cases: $\Psi_{P I D}^{i j} \approx 0 \mathrm{rad}$ and $\Psi_{P I D}^{j i} \approx 1.5 \mathrm{rad}$ or vice versa: $\Psi_{P I D}^{j i} \approx 0 \mathrm{rad}$ and $\Psi_{P I D}^{i j} \approx 1 \mathrm{rad}$. This is consistent with the results in Fig. S2, as one residue donors a backbone, and the second residue donors a sidechain. These distributions are, however, very broad and range for distances $5.5 \AA<\mathrm{r}<7 \AA$ (and become even broader for larger distances, see Fig. S7).

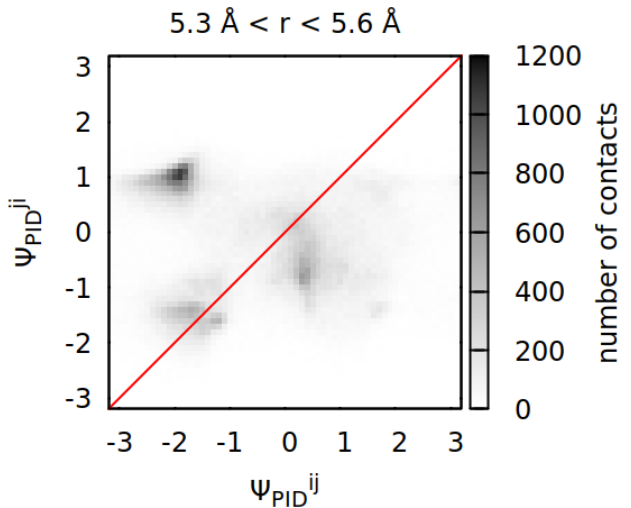

backbone-sidechain (bs)

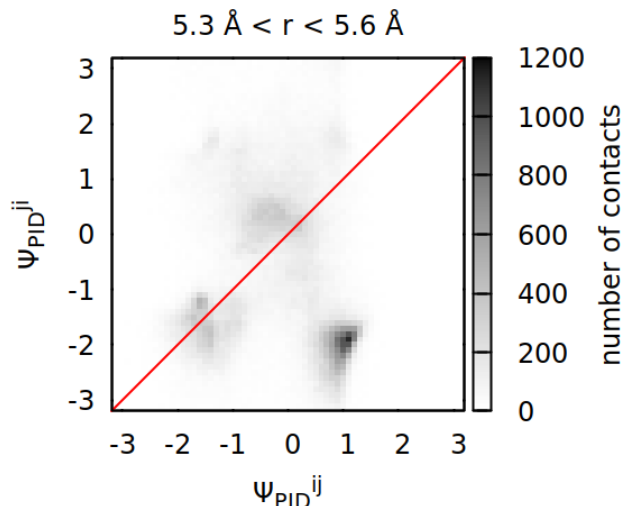

sidechain-backbone (sb)

Figure S5: Two-dimensional distribution of contacts, where the first PID angle (in radians) in a contact is on one axis and the second angle is on the other axis. Those contacts could also include other types of overlaps (bb or ss). $\mathrm{C}_{\alpha}-\mathrm{C}_{\alpha}$ distance $r$ is within the range given on top of the graphs.

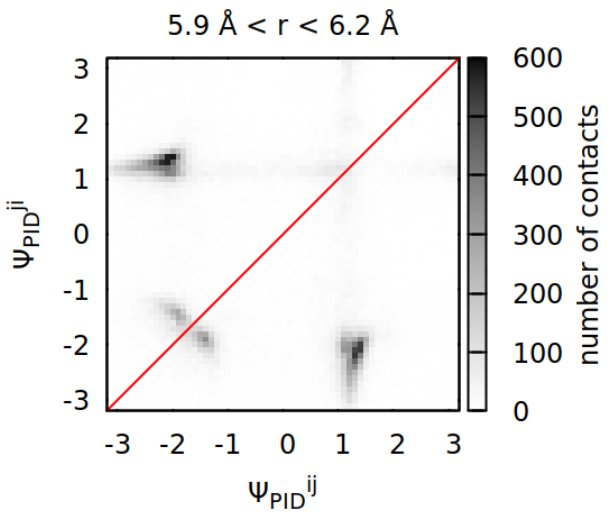

only backbone-backbone (bb)

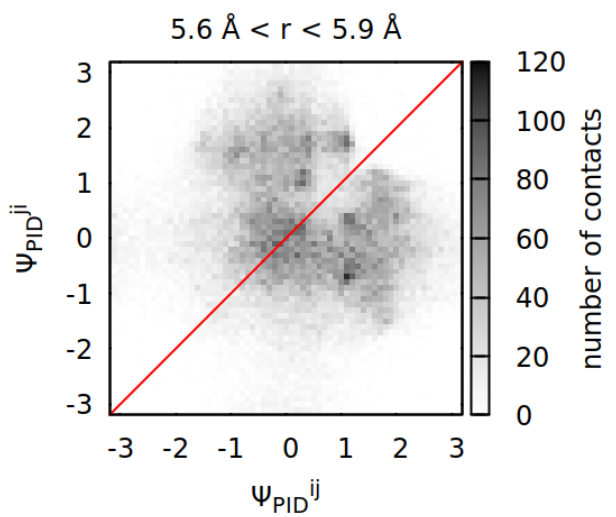

only sidechain with backbone (bs and sb)

Figure S6: Two-dimensional distribution of contacts, where the first PID angle (in radians) in a contact is on one axis and the second angle is on the other axis. Contacts that contain only bb overlaps (left panel) or only bs and/or sb overlaps (right) were included. $\mathrm{C}_{\alpha}-\mathrm{C}_{\alpha}$ distance $r$ is within the range given on top of the graphs. 


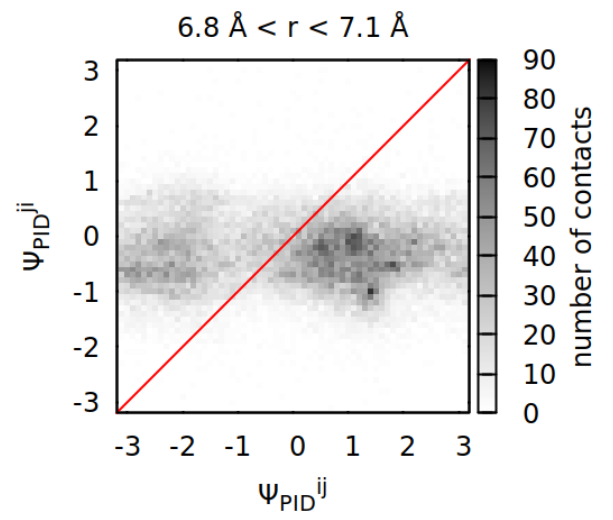

only backbone-sidechain (bs)

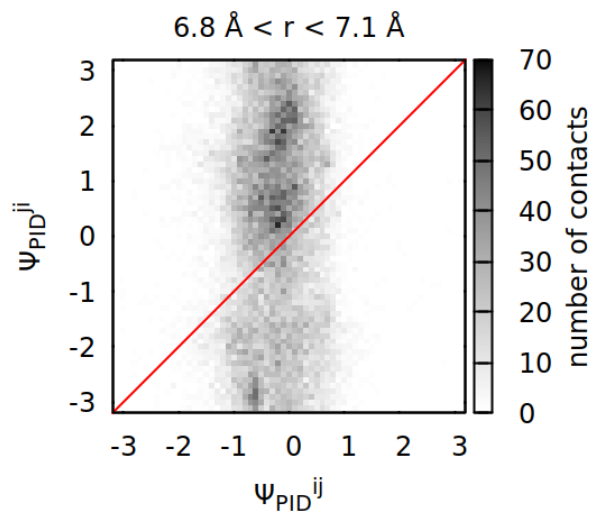

only sidechain-backbone $(\mathrm{sb})$

Figure S7: Two-dimensional distribution of contacts, where the first PID angle (in radians) in a contact is on one axis and the second angle is on the other axis. Contacts that contain only bs overlaps (left panel) or only sb overlaps (right) were included. $\mathrm{C}_{\alpha}-\mathrm{C}_{\alpha}$ distance $r$ is within the range given on top of the graphs. Note that the number of contacts is smaller than in all other distributions.

\subsection{Statistics without dividing into bb, bs and ss types}

The bs contacts were hard to quantify, so total distributions were also plotted (Fig. S8). It turns out that $\mathrm{bb}$ and ss contacts have pretty distinguishable peaks even without using the information about the contact type from the overlaps. The top panels of Fig. S8 show that bb contacts have two peaks corresponding to $\psi_{0}^{b b+}=1.05 \mathrm{rad}$ and $\psi_{0}^{b b-}=-1.44 \mathrm{rad}$, while ss contacts correspond to one broader $\psi_{0}^{s s}=-0.23 \mathrm{rad}$ peak (shown on the bottom panel). Those are the same peaks as in Fig. S2.

It is interesting to note that $\psi_{P I D} \approx+1 \mathrm{rad}$ is common for smaller $\mathrm{C}_{\alpha}-\mathrm{C}_{\alpha}$ distance than those for $\psi_{P I D} \approx-1 \mathrm{rad}$, which is reflected in our potential $\left(r_{\min }^{b b+}=r_{\min }^{b b-}-0.6 \AA\right)$.
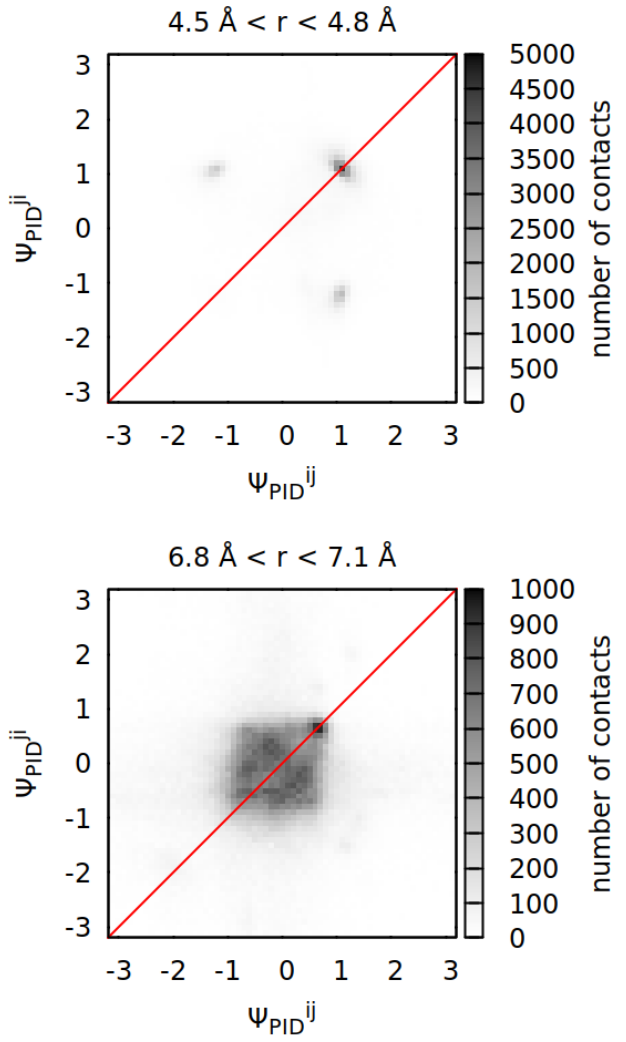

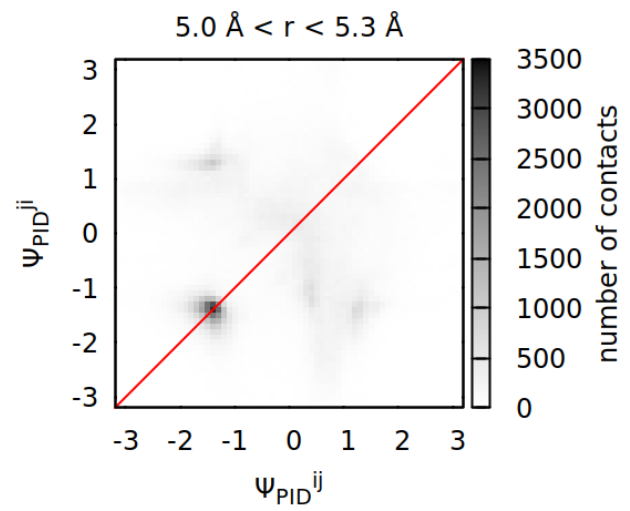

Figure S8: Two-dimensional distribution of contacts, where the first PID angle (in radians) in a contact is on one axis and the second angle is on the other axis. 


\section{The parameterization}

\subsection{Proteins used for the parameterization}

We used a set of 23 intrinsically disordered proteins with different lengths, occupying different areas of the Das-Pappu diagram (see Fig. S9) and the Uversky plot (see Fig. S10) [6]. Full Das-Pappu diagram S11 shows the whole state space with unused regions (proteins usually do not have that many charged residues [7]).

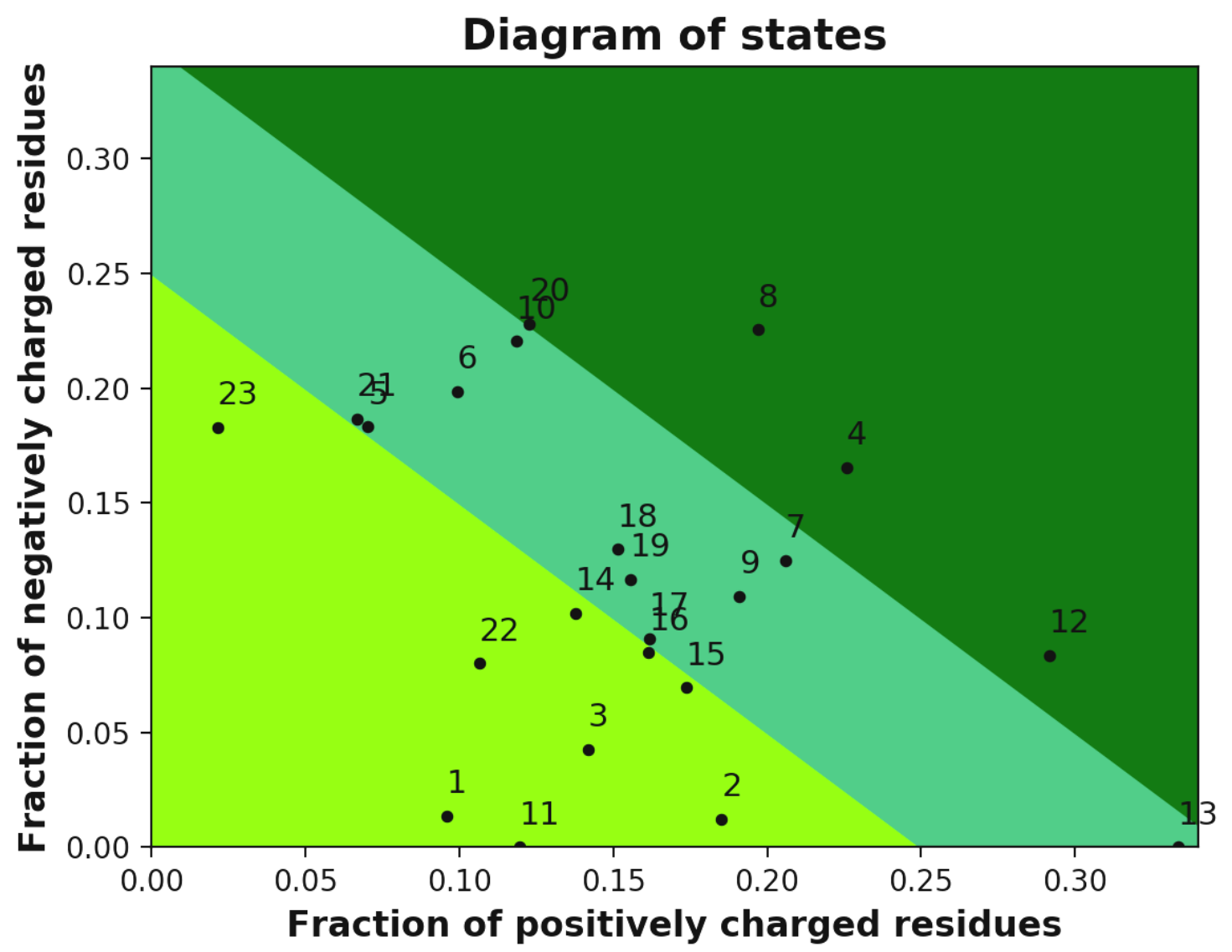

Figure S9: Das-Pappu diagram of the 23 IDPs used for the parameterization, labeled by their number in Table S2.

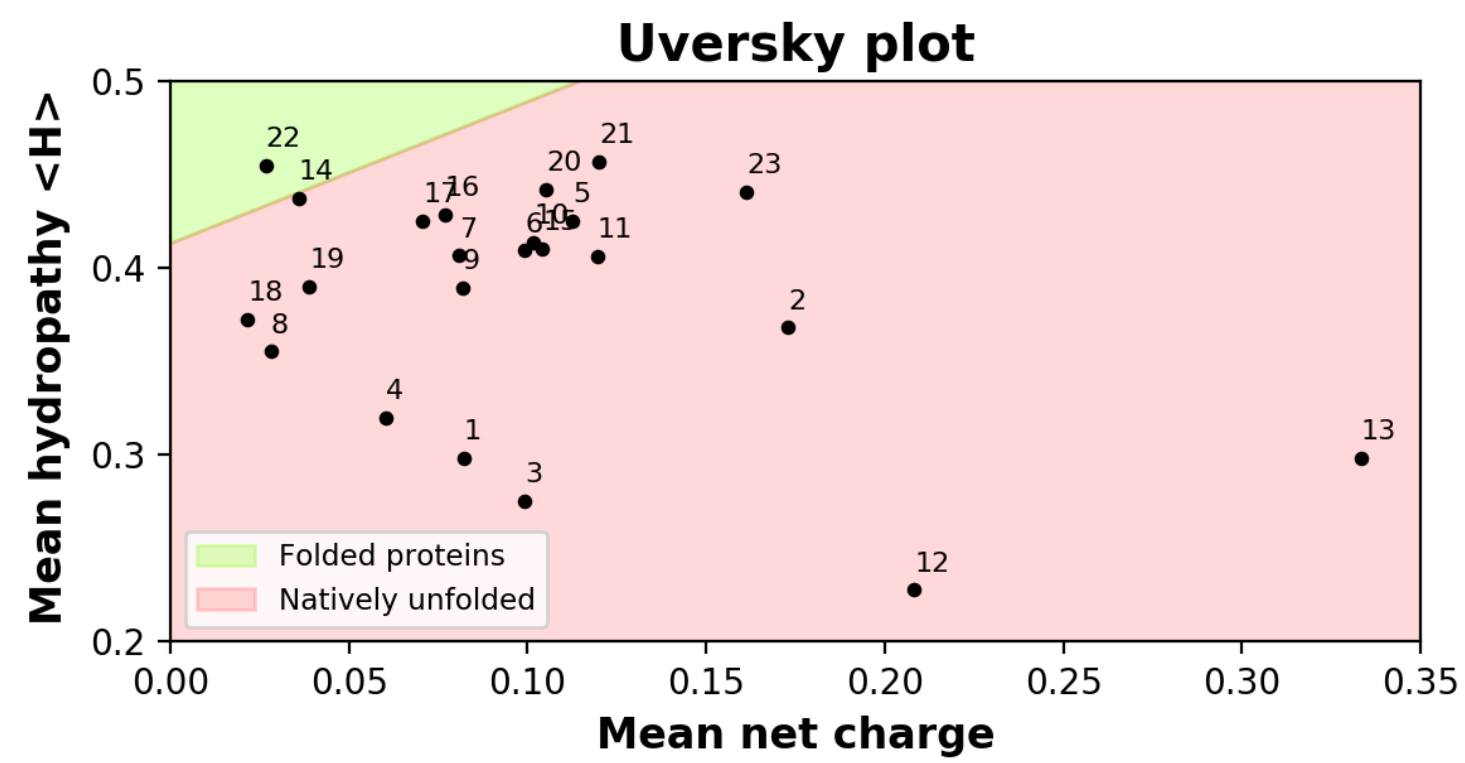

Figure S10: Uversky plot of the 23 IDPs used for the parameterization, labeled by their number in Table S2. The vertical axis uses Kyte-Doolittle hydropathy score [8] rescaled to 0-1 range. 
It is interesting to note that the value of the screening length used significantly affects the results. Using uniform $s=10 \AA$ resulted in worse agreement with experiment (we were unable to find a model better than Gaussian chain with $b=6.7 \AA$, results not shown).

\begin{tabular}{|r|r|r|r|r|r|}
\hline nr & id & $n$ & $R_{g} / \AA$ & $s / \AA$ & source \\
\hline 1 & IB5 & 73 & $27.9 \pm 1.0$ & 13.6 & {$[9]$} \\
\hline 2 & Ash1 & 81 & $28.4 \pm 3.4$ & 7.9 & {$[9]$} \\
\hline 3 & II1ng & 141 & $41.1 \pm 1.0$ & 13.6 & {$[9]$} \\
\hline 4 & RNaseE & 248 & $52.6 \pm 0.3$ & 7.85 & {$[9]$} \\
\hline 5 & ACTR & 71 & $25.1 \pm 1.3$ & 6.8 & {$[10]$} \\
\hline 6 & NHE1 & 131 & $36.3 \pm 1.8$ & 6.8 & {$[10]$} \\
\hline 7 & sNase & 136 & $21.2 \pm 1.0$ & 23.3 & {$[10]$} \\
\hline 8 & 5 AAA & 142 & $22.15 \pm 0.87$ & 4.3 & {$[11]$} \\
\hline 9 & 6 AAA & 110 & $28.1 \pm 0.1$ & 7.7 & {$[11]$} \\
\hline 10 & 8 AAC & 59 & $14.6 \pm 0.5$ & 13.6 & {$[11]$} \\
\hline 11 & 9 AAA & 92 & $29.9 \pm 0.3$ & 7.55 & {$[11]$} \\
\hline 12 & his5 & 24 & $13.6 \pm 0.2$ & 7.85 & {$[12]$} \\
\hline 13 & RS & 24 & $12.62 \pm 0.07$ & 6.7 & {$[13]$} \\
\hline 14 & tauK10 & 168 & $40.0 \pm 1.0$ & 7.4 & {$[14,19]$} \\
\hline 15 & tauK17 & 144 & $36.0 \pm 2.0$ & 7.4 & {$[14,19]$} \\
\hline 16 & tauK18 & 130 & $38.0 \pm 3.0$ & 7.4 & {$[14,19]$} \\
\hline 17 & tauK19 & 99 & $35.0 \pm 1.0$ & 7.4 & {$[14,19]$} \\
\hline 18 & tauK25 & 185 & $41.0 \pm 2.0$ & 7.4 & {$[14,19]$} \\
\hline 19 & tauK44 & 283 & $52.0 \pm 2.0$ & 7.4 & {$[14,19]$} \\
\hline 20 & RNF4 & 57 & $25.8 \pm 3.9$ & 8.5 & {$[15,19]$} \\
\hline 21 & NRG1 & 75 & $26.8 \pm 1.1$ & 7.4 & {$[16,19]$} \\
\hline 22 & PIR & 75 & $26.5 \pm 0.5$ & 7.8 & {$[17,19]$} \\
\hline 23 & p53 & 93 & $28.7 \pm 0.3$ & 6.6 & {$[18,19]$} \\
\hline & & & & \\
\hline
\end{tabular}

Table S2: Properties of the 23 IDPs used for the parameterization: id used for identification, number of residues $n$, experimental $R_{g}$, screening length $s$ and source of information.

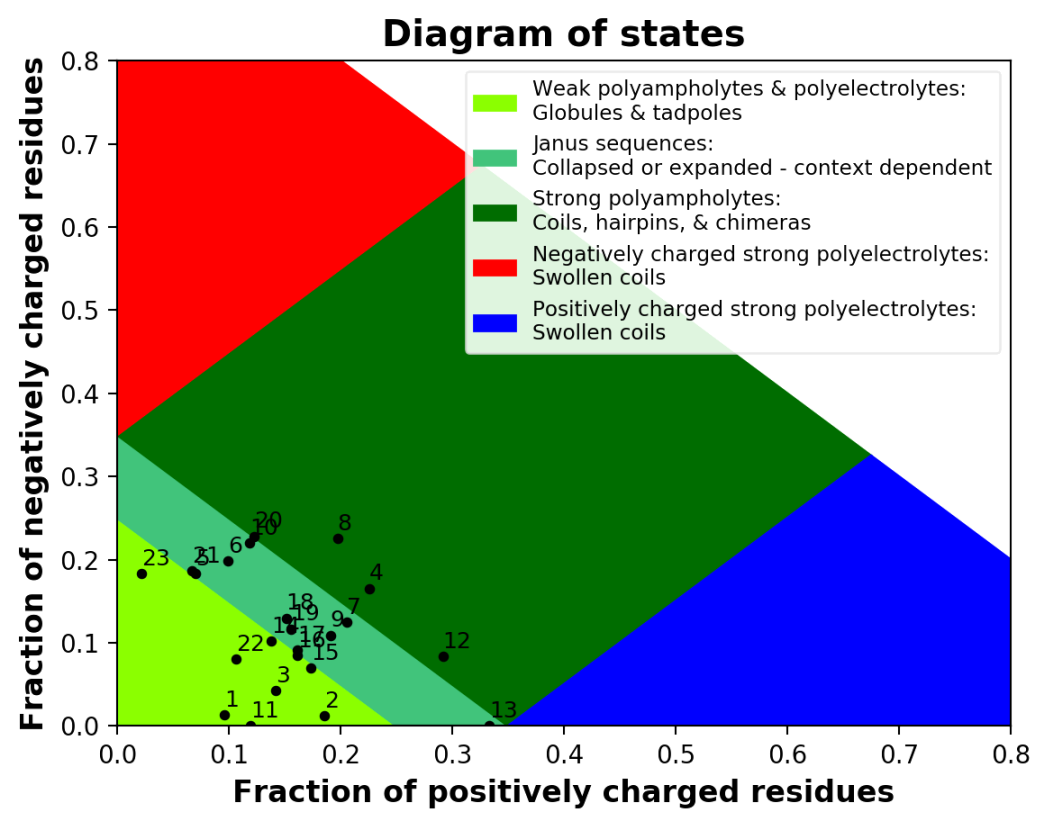

Figure S11: Das-Pappu diagram of the 23 IDPs, labeled by their number in Table S2. The shape of Janus sequences depends on factors like the ionic force. In our simulations we always assumed the same ionic force as in SAXS experiments. 
IB 5|SARSP P GKP QGP P QQEGNKP QGP P P P GKP QGP P P AGGNP QQP QAP P AGKP QGPPPPPQGGRPPRPAQGQQPPQ

Ash1GASASS SP SP STP TKSGKMRS S SPVRP KAYTP SP RSP NYHRFALDSPPQS PRRSSNSSITKKGSRRSSGSSPTRHTTRVCV

I I 1 ngI S GKPVGRRP QGGNQP QRP P P P P GKP QGP P P QGGWQSQGP P P P P GKP EGR P P QGRNQS QGP P P HP GKP ERP P P QGGS QGTP P P P GKP ERP P P QGGNQS HR PPPPP GKP ERPP P QGGNQSRGP P P HRGKP EGP PPQEGNKSR

RNa s EERRQDRRKP RQNNRRDRNERRDTRSERTEGSDNREENRRNRRQAQQQTAE TRESRQQAEKARIADTADEQQAPRRERSRRRNDDKRQAQQEAKALNVEEQ SVQETEQEERVRPVQPRRKQRQLNQKVRYEQSVAEEAVVAPVVEETVAAE P IVQEAPAPRTELVKVP LPVVAQTAPEQQEENNADNRDNGGMP RRSRRSP RHLRVSGQRRRRYRDCRYP IQSPMP LTVACASPELAS GKVWIRVP IVR

ACTRGTQNRP LLRNS LDDLVGP P SNLEGQSDERALLDQLHTLLSNTDATGLEE I DRALGIPELVNQGQALEPKQD

NHE 1MVPAHKLDSP TMSRARIGSDP LAYEPKEDLPVITIDPASP QSPESVDLVN EELKGKVLGLSRDPAKVAEEDEDDDGG IMMRSKETSSP GTDDVFTPAP SD SP SSQRIQRCLSDP GPHPEP GEGEPFFPKGQ

SNa S AATSTKKLHKEPATLIKAIDGDTVKLMYKGQPMTFRLLLVDTPETKHPKKG VEKYGPEASAFTKKMVENAKKIEVEFDKGQRTDKYGRGLAYIYAD GKMVN EALVRQGLAKVAYVYKPNNTHEQHLRKSEAQAKKEK

5AAAMDYKDDDDKNRALSPMVSEFET IEQENSYNEWLRAKVATSLADPRPA IPH DEVERRMAERFAKMRKERSKQMDYKDDDDKNRALSPMVSEFET IEQENSY NEWLRAKVAT SLADPRPAIP HDEVERRMAERFAKMRKERSKQ

6AAAVRTKADSVP GTYRKVVAARAP RKVLGS STSATNS TSVSSRKAENKYAGGN PVCVRP TP KWQKGIGEFFRLSP KDSEKENQIPEEAGSSGLGKAKRKACP L QPDHTNDEKE

8AACMEAIAKHDF SATADDELSFRKTQ I LK I LNMEDD SNWTRAELDGKEGLIPS NYIEMKNHD

9AAAGSMTP STP PRSRGTRYLAQP S GNT S S SALMQGQKTPQKP SQNLVPVTP ST TKSFKNAP LLAP PNSNMGMT SP FNGLT SPQRSPFP KSSVKRT

his 5DSHAKRHHGYKRKFHEKHHSHRGY

RSGAMGP SYGRSRSRSRSRSRSRSRS

tauK10QTAPVPMP DLKNVKSKIGSTENLKHQP GGGKVQIVYKPVDLSKVTSKCGS LGN I HHKP GGGQVEVKSEKLDF KDRVQSKIGS LDNITHVP GGGNKKIETH KLTFRENAKAKTDHGAEIVYKSPVVSGDTSPRHLSNVSSTGS IDMVDSPQ LATLADEVSASLAKQGL

tauK1 7SSP GSP GTP GSRSRTP SLPTPP TREPKKVAVVRTPPKSP SSAKSRLQTAP VPMP DLKNVKSKIGS TENLKHQP GGGKVQIVYKPVDLSKVTSKCGSLGNI HHKP GGGQVEVKSEKLDFKDRVQSKI GSLDNITHVP GGGNKKIE

tauK1 8MQTAPVPMP DLKNVKSKI GS TENLKHQP GGGKVQ I INKKLDLSNVQSKCG SKDNI KHVP GGGSVQ IVYKPVDLSKVT SKCGS LGN I HHKP GGGQVEVKSE KLDEKDRVQSKIGSLDNITHVP GGGNKKIE

tauK1 9MQTAPVPMP DLKNVKSKI GSTENLKHQP GGGKVQIVYKPVDLSKVTSKCG SLGN I HHKP GGGQVEVKSEKLDFKDRVQSKI GS LDNI THVP GGGNKKIE

tauK2 5MAEP RQEFEVMEDHAGTYGLGDRKDQGGYTMHQDQEGDTDAGLKAEEAGI GDTP S LEDEAAGHVTQARMVSKSKDGTGSDDKKAKGADGKTKIATPRGAA PPGQKGQANATRIPAKTPPAPKTPP S S GEPP KSGDRSGYSSP GSP GTP GS RSRTP SLPTPPTREPKKVAVVRTPPKSP SSAKSRL

tauK4 4MAEP RQEFEVMEDHAGTYGLGDRKDQGGYTMHQDQEGDTDAGLKAEEAGI GDTP S LEDEAAGHVTQARMVSKSKDGTGSDDKKAKGADGKTKIATPRGAA PP GQKGQANATRIPAKTPPAPKTPP SSGEPPKSGDRS GYSSP GSP GTPGS RSRTP SLP TPP TREP KKVAVVRTPPKSP SSAKSRLQTAPVPMPDLKNVKS KIGSTENLKHQP GGGKVQIVYKPVDLSKVTSKCGSLGN I HHKP GGGQVEV KSEKLDFKDRVQSKIGSLDNITHVP GGGNKKIE

RNF 4 GHMGSWEAEP IELVETAGDE IVDLTCES LEPVVVDLTHNDSVVIVDERRR PRRNARR

NRG1MEIYSP DMSEVAAERSS SP STQLSADP S LDGLPAAEDMP EP QTEDGRTPG LVGLAVPCCACLEAERLRGCLNSEK

P IRQSVSPMRSVSENSLVAMDF SGQKTRVIDNP TEALSVAVEEGLAWRKKGCL RLGNHGSP TAP SQSSAVNMALHRSQ

p53MEEP QSDP SVEP P LSQETF SDLWKLLPENNVLSP LP S QAMDDLMLSP DD I EQWF TEDP GP DEAPRMPEAAP PVAPAPAAP TPAAPAPAP SWP L

Table S3: Sequences of the 23 IDPs used for the parameterization. 


\subsection{Model ranking}

We considered 246 different versions of our model. All symbols used in their names are explained in the legend (Table S4). A few of them were not defined in the main text: we considered versions (indicated by letter W) where the PID angle potential was fitted to the Boltzmann inversion potential based on all overlap contacts (green histograms in Fig. 2 in the main text).

We also checked L-J potential with a bump (indicated by a subscript ${ }_{B}$ ), designed to mimic the potential barrier resulting from a hydration shell made by water molecules surrounding the residues.

In charged residues the charge is located near the end of the sidechain, so we tried using the PID potential for the electrostatic interactions (denoted by letter $\mathrm{D}$ ) to take account of that directionality.

The best fit to the experimental data for the Kuhn length of the Gaussian chain is $b=6.7 \AA$, but we also included other values of $b$ for comparison.

In the Table S5 we list the models sorted by their Pearson coefficient $P$ (see the main text). We also included $\chi^{2}=\frac{1}{N} \sum_{p=1}^{N}\left(\frac{\left(R_{g}^{e x p}-R_{g}^{s i m}\right)^{2}}{\sigma_{s i m}^{2}+\sigma_{\exp }^{2}}\right)_{p}$, where $\sigma_{\text {sim }}$ is the uncertainty of the simulation result defined by the jacknife resampling method and $\sigma_{\text {exp }}$ is the experimental uncertainty from Table S2. Uncertainties are much smaller than $R_{g}^{e x p}-R_{g}^{s i m}$, so the values of $\chi^{2}$ are large (values bigger than 1000 are indicated as ,>1000").

For each protein and each version of the model we made 20 trajectories. For the quasi-adiabatic models (letter A) the equilibration time was $75000 \tau$ and the total simulation time was $150000 \tau$. For PID models (letters $\mathrm{P}$ and W) both times were 10x smaller (based on the times needed for $R_{g}$ to stop depending on the initial conditions). This difference may indicate that the timescales in the PID model are longer than in the previous one, so Fig. 6 in the main text may underestimate the efficiency of the PID model.

\begin{tabular}{|c|c|}
\hline $\mathrm{P}$ & PID potential (fit to red histograms in Fig. 2) \\
\hline A & Quasi-adiabatic potential \\
\hline W & PID potential, wide version (fit to green histograms in Fig. 2) \\
\hline superscript ${ }^{+}$ & $i, i+4$ attractive contacts on \\
\hline superscript ${ }^{-}$ & $i, i+4$ attractive contacts off \\
\hline $\mathrm{L}$ & Standard Lenard-Jones potential \\
\hline $\mathrm{F}$ & Lenard-Jones potential with flat region between $r_{m i n}^{b b}$ and $r_{m i n}^{s s}$ for ss contacts \\
\hline $\mathrm{L}_{B}$ & potential defined by equation $\phi(r)=\epsilon^{L J}\left[\left(\frac{r_{\min }}{r}\right)^{12}-\frac{9}{2^{1 / 6}}\left(\frac{r_{\min }}{r}\right)^{7}+\frac{13}{2}\left(\frac{r_{\min }}{r}\right)^{6}\right]$ \\
\hline $\mathrm{F}_{B}$ & Same as $\mathrm{L}_{B}$, but with flat region between $r_{\min }^{b b}$ and $r_{\min }^{s s}$ for ss contacts \\
\hline $\mathrm{ME}$ & Matrix of interactions where each contact has the same amplitude (default $1 \epsilon$ ) \\
\hline MJ & Miyazawa-Jernigan matrix of interactions [3] \\
\hline MD & MDCG matrix of interactions [4] \\
\hline Subscript $0-1$ & scaling factor for the matrix (from ${ }_{0}$ to ${ }_{1}$ ) \\
\hline $\mathrm{C}$ & Classic Debye-Hueckel electrostatics with permittivity 80 \\
\hline $\mathrm{T}$ & Debye-Hueckel electrostatics with permittivity $4 \AA / r[5]$ \\
\hline $\mathrm{R}$ & $\begin{array}{l}\text { Electrostatics only for residues with the same sign } \\
\text { (oppositely charged residues interact via the ss contacts as uncharged residues) }\end{array}$ \\
\hline $\mathrm{D}$ & $\begin{array}{l}\text { Directional electrostatics with PID potential } \lambda_{A}\left(\psi_{A}\right) \lambda_{B}\left(\psi_{B}\right) V_{D-H}(r) \text {, } \\
\text { where } V_{D-H}(r)=\frac{ \pm e^{2} \exp (-r / s)}{4 \pi \varepsilon \varepsilon_{0} r} \text { is the classic Debye-Hueckel potential }\end{array}$ \\
\hline $\mathrm{GC}$ & Gaussian chain model with Kuhn length b \\
\hline
\end{tabular}

Table S4: Legend for the names of the model variants. 


\begin{tabular}{|c|c|c|c|c|c|}
\hline 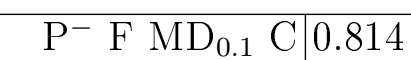 & 3.3 & $\mathrm{P}^{+} \mathrm{F}_{B} \mathrm{MD}_{0.1} \mathrm{C} \mid 0.726$ & 40.1 & $\mathrm{P}^{-} \mathrm{F}_{B} \mathrm{MD}_{0.3} \mathrm{C} \mid 0.682$ & 72 \\
\hline 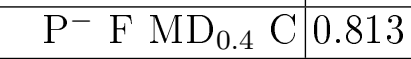 & 14.0 & 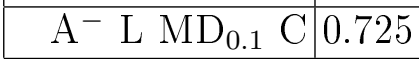 & 99.8 & $\mathrm{~W}^{-} \mathrm{F}_{B} \mathrm{MD}_{0.2} \mathrm{C} \quad 0.681$ & 138.5 \\
\hline 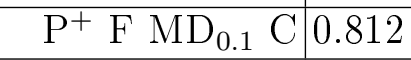 & 13.7 & \begin{tabular}{l|l|l}
$\mathrm{A}^{-} \mathrm{LD}_{0.4} \mathrm{C}$ & 0.725 \\
\end{tabular} & 99.5 & $\mathrm{~W}^{+} \mathrm{F}_{B} \mathrm{MD}_{0.1} \mathrm{C} \quad 0.679$ & 156.6 \\
\hline $\mathrm{P}^{-} \mathrm{F} \mathrm{ME}_{0.0} \mathrm{C} 0.812$ & 14.4 & 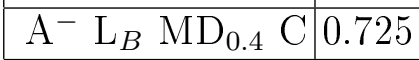 & 99.5 & $\mathrm{P}^{+} \mathrm{F}_{B} \mathrm{MD}_{1} \mathrm{~T} 0.679$ & $>1000$ \\
\hline 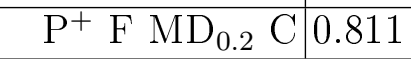 & 14.3 & 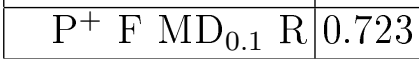 & 21.2 & 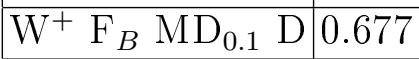 & 147 \\
\hline $\mathrm{P}^{-} \mathrm{F} \mathrm{ME}_{0.0} \mathrm{~T} 0.809$ & 11.6 & \begin{tabular}{|l|l|l}
$\mathrm{V}^{+}$ & $\mathrm{MD}_{0.1} \mathrm{R}$ & 0.723 \\
\end{tabular} & 130.0 & $\mathrm{P}^{+} \mathrm{F}_{B} \mathrm{MD}_{0.1} \mathrm{C} 0.677$ & 32.9 \\
\hline $\begin{array}{l}\mathrm{P}^{-} \mathrm{F} \mathrm{MD}_{0.2} \mathrm{C} \\
0.809\end{array}$ & 13.9 & 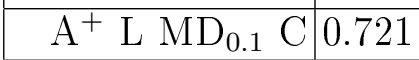 & 137.0 & $\mathrm{P}^{+} \mathrm{F}_{B} \mathrm{MD}_{0.2} \mathrm{C} 0.675$ & 32.2 \\
\hline 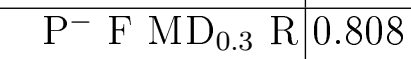 & 29.6 & 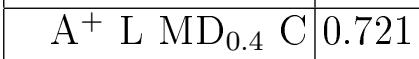 & 136.7 & $\mathrm{~W}^{+} \mathrm{F}_{B} \mathrm{MD}_{0.3} \mathrm{R} 0.674$ & 173.5 \\
\hline $\mathrm{P}^{+} \mathrm{F} \mathrm{MD}_{0.4} \mathrm{C} 0.806$ & 16.7 & 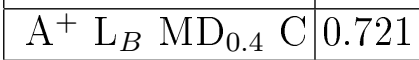 & 136.7 & $\mathrm{~A}^{+} \mathrm{F} \mathrm{MD}_{0.2} \mathrm{R} 0.673$ & 197.3 \\
\hline $\mathrm{P}^{+} \mathrm{F} \mathrm{MD}_{0.4} \mathrm{D} 0.803$ & 13.6 & 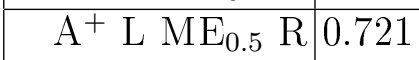 & 80.7 & $\mathrm{~A}^{+} \mathrm{F}_{B} \mathrm{MD}_{0.2} \mathrm{R} 0.673$ & 97.3 \\
\hline 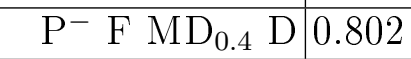 & 10.7 & 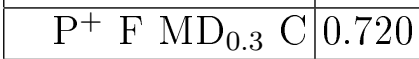 & 113.1 & $\mathrm{~A}^{+} \mathrm{F} \mathrm{MD}_{0.1} \mathrm{R} 0.673$ & 197.5 \\
\hline $\mathrm{P}^{+} \mathrm{F} \mathrm{MD}_{0.1} \mathrm{D} 0.799$ & 13.0 & $\mathrm{P}^{+} \mathrm{F}_{B} \mathrm{MD}_{0.3} \mathrm{C} \mid 0.720$ & 27.0 & $\mathrm{~A}^{+} \mathrm{F}_{B} \mathrm{MD}_{0.1} \mathrm{R} 0.673$ & 197.5 \\
\hline 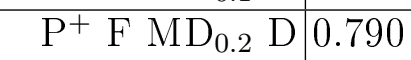 & 13.1 & 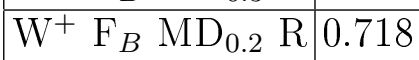 & 136.4 & $\mathrm{~A}^{+} \mathrm{F} \mathrm{MD}_{0.4} \mathrm{R} 0.673$ & 202.1 \\
\hline $\mathrm{GC}, b=6.7 \AA 0.788$ & 89.1 & 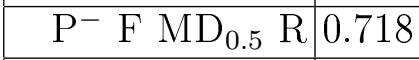 & 386.9 & $\mathrm{~A}^{+} \mathrm{F}_{B} \mathrm{MD}_{0.4} \mathrm{R} 0.673$ & 202.1 \\
\hline $\mathrm{P}^{-} \mathrm{F}_{B} \mathrm{MD}_{0.5} \mathrm{C} 0.788$ & 17.9 & \begin{tabular}{l|l|l}
$\mathrm{P}^{-} \mathrm{F} \mathrm{MD}_{0.1} \mathrm{R}$ & 0.7
\end{tabular} & 386.9 & 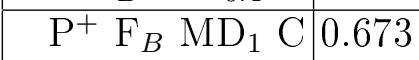 & 1000 \\
\hline $\mathrm{P}^{-} \mathrm{F}_{B} \mathrm{MD}_{0.1} \mathrm{C} 0.788$ & 17.9 & 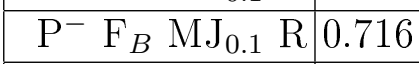 & 24.2 & $\mathrm{~W}^{-} \mathrm{F}_{B} \mathrm{MD}_{0.4} \mathrm{D} 0.673$ & 125.2 \\
\hline $\mathrm{P}^{-} \mathrm{F}_{B} \mathrm{MJ}_{0.1} \mathrm{C} 0.786$ & 17.3 & \begin{tabular}{|l|l}
$\mathrm{P}^{+} \mathrm{F}_{B} \mathrm{MD}_{1} \mathrm{R}$ & 0.713
\end{tabular} & 525.2 & $\mathrm{P}^{+} \mathrm{F}_{B} \mathrm{MD}_{0.2} \mathrm{D} 0.672$ & \\
\hline $\mathrm{P}^{-} \mathrm{F} \mathrm{ME}_{0.0} \mathrm{D} 0.786$ & 13.6 & 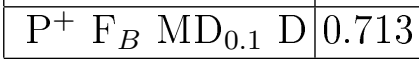 & 29.0 & $\mathrm{P}^{+} \mathrm{F}_{B} \mathrm{MD}_{0.1} \mathrm{D} 0.672$ & 27.7 \\
\hline $\mathrm{P}^{-} \mathrm{F} \mathrm{MD}_{0.1} \mathrm{D} 0.786$ & 13.4 & 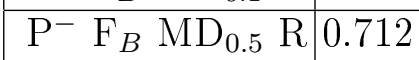 & 24.5 & $\mathrm{~W}^{+} \mathrm{F}_{B} \mathrm{MD}_{0.2} \mathrm{D} 0.670$ & .0 \\
\hline $\mathrm{P}^{-} \mathrm{F}_{B} \mathrm{ME}_{0.1} \mathrm{C} 0.785$ & 61.6 & 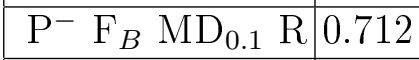 & 24.5 & $\mathrm{~W}^{-} \mathrm{F}_{B} \mathrm{MD}_{0.1} \mathrm{D} \quad 0.669$ & 123.0 \\
\hline $\mathrm{P}^{-} \mathrm{F} \mathrm{MD}_{0.2} \mathrm{D} 0.783$ & 12.5 & 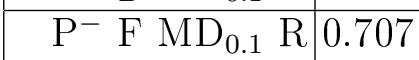 & 25.2 & $\mathrm{P}^{+} \mathrm{F}_{B} \mathrm{MD}_{0.4} \mathrm{C} 0.669$ & 34.0 \\
\hline $\mathrm{P}^{+} \mathrm{F} \mathrm{MD}_{0.3} \mathrm{R} 0.783$ & 43.1 & 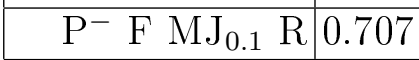 & 333.2 & $\mathrm{~W}^{-} \mathrm{F}_{B} \mathrm{MD}_{0.2} \mathrm{D} 0.668$ & 127.7 \\
\hline $\mathrm{A}^{-} \mathrm{L} \mathrm{MD}_{0.4} \mathrm{R} 0.765$ & 62.8 & 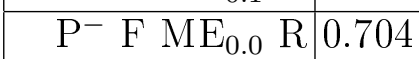 & 27.6 & $\mathrm{P}^{-} \mathrm{F}_{B} \mathrm{MD}_{0.1} \mathrm{C} 0.666$ & 34.4 \\
\hline $\mathrm{A}^{-} \mathrm{L} \mathrm{MD}_{0.1} \mathrm{R} \quad 0.764$ & 62.5 & \begin{tabular}{l|l|l}
$\mathrm{P}^{-} \mathrm{F} \mathrm{MD}_{0.2} \mathrm{R}$ & 0.702 \\
\end{tabular} & 25.9 & 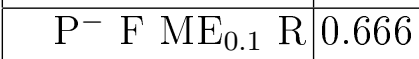 & 677.3 \\
\hline $\mathrm{A}^{+} \mathrm{L} \mathrm{MD}_{0.4} \mathrm{R} 0.752$ & 94.2 & 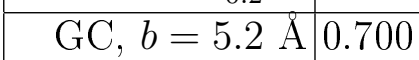 & 513.4 & $\mathrm{P}^{+} \mathrm{F}_{B} \mathrm{MD}_{0.3} \mathrm{R} 0.665$ & 35.1 \\
\hline $\mathrm{A}^{+} \mathrm{L}_{B} \mathrm{MD}_{0.4} \mathrm{R} 0.752$ & 95.0 & 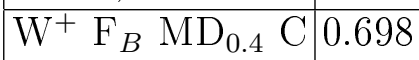 & 144. & $\mathrm{P}^{+} \mathrm{F}_{B} \mathrm{MD}_{0.4} \mathrm{D} 0.663$ & 29.8 \\
\hline $\mathrm{A}^{+} \mathrm{L} \mathrm{MD}_{0.1} \mathrm{R} 0.751$ & 95.3 & \begin{tabular}{l|l|l}
$\mathrm{P}^{+} \mathrm{F} \mathrm{MD}_{0.5} \mathrm{R}$ & 0.698
\end{tabular} & 211 & $\mathrm{P}^{-} \mathrm{F}_{B} \mathrm{MD}_{0.1} \mathrm{D} 0.663$ & 33.8 \\
\hline $\mathrm{P}^{-} \mathrm{F}_{B} \mathrm{ME}_{0.1} \mathrm{R} 0.744$ & 19.8 & 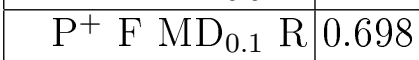 & 211 & $\mathrm{P}^{-} \mathrm{F}_{B} \mathrm{MD}_{0.4} \mathrm{C} 0.659$ & 35.9 \\
\hline $\mathrm{W}^{-} \mathrm{F}_{B} \mathrm{MD}_{0.4} \mathrm{R} 0.744$ & 97.0 & \begin{tabular}{|l|l|l}
$\mathrm{W}^{-} \mathrm{F}_{B} \mathrm{MD}_{0.4} \mathrm{C}$ & 0.698
\end{tabular} & 118.2 & $\mathrm{P}^{-} \mathrm{F}_{B} \mathrm{MD}_{0.2} \mathrm{C} 0.658$ & 35.1 \\
\hline $\mathrm{P}^{-} \mathrm{F} \mathrm{MD}_{0.3} \mathrm{C} 0.738$ & 214.6 & 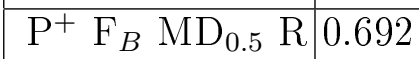 & 34.1 & $\mathrm{P}^{-} \mathrm{F}_{B} \mathrm{MD}_{0.3} \mathrm{R} 0.656$ & 9 \\
\hline $\mathrm{P}^{-} \mathrm{F}_{B} \mathrm{MD}_{0.3} \mathrm{C} 0.733$ & 24.1 & 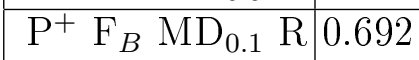 & 34.1 & $\mathrm{P}^{-} \mathrm{F} \mathrm{MD}_{0.5} \mathrm{C} 0.655$ & 629.9 \\
\hline $\mathrm{W}^{-} \mathrm{F}_{B} \mathrm{MD}_{0.2} \mathrm{R} 0.730$ & 101.2 & \begin{tabular}{|l|l|l}
$\mathrm{W}^{-} \mathrm{F}_{B}$ & $\mathrm{MD}_{0.3} \mathrm{R}$ & 0.688
\end{tabular} & 127.8 & $\mathrm{P}^{-} \mathrm{F} \mathrm{MD}_{0.1} \mathrm{C} 0.655$ & 629.9 \\
\hline $\mathrm{W}^{-} \mathrm{F}_{B} \mathrm{MD}_{0.1} \mathrm{R} 0.730$ & 79.6 & 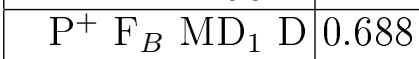 & 689 & $\mathrm{P}^{-} \mathrm{F}_{B} \mathrm{MD}_{0.2} \mathrm{D} 0.653$ & 34.1 \\
\hline 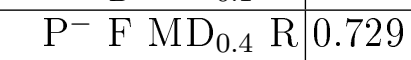 & 21.2 & 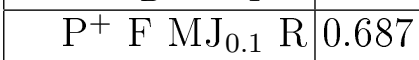 & 254.1 & $\mathrm{P}^{-} \mathrm{F}_{B} \mathrm{MD}_{0.4} \mathrm{D} 0.651$ & 35.8 \\
\hline $\mathrm{P}^{-} \mathrm{F}_{B} \mathrm{MD}_{0.5} \mathrm{R} 0.728$ & 183.6 & \begin{tabular}{|l|l|l}
$\mathrm{W}^{-} \mathrm{F}_{B} \mathrm{MD}_{0.1} \mathrm{C}$ & 0.686 \\
\end{tabular} & 99.0 & $\mathrm{~A}^{-} \mathrm{F} \mathrm{MD}_{0.1} \mathrm{R} \quad 0.650$ & 167.4 \\
\hline $\mathrm{P}^{-} \mathrm{F}_{B} \mathrm{MD}_{0.3} \mathrm{R} 0.728$ & 483.6 & 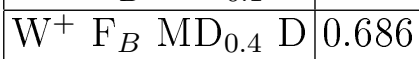 & 148.9 & $\mathrm{~A}^{-} \mathrm{F} \mathrm{MD}_{0.4} \mathrm{R} 0.649$ & 167.3 \\
\hline $\mathrm{P}^{+} \mathrm{F}_{B} \mathrm{MD}_{0.1} \mathrm{~T} 0.727$ & 29.9 & 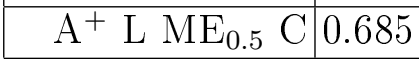 & 100.2 & $\mathrm{~A}^{-} \mathrm{F}_{B} \mathrm{MD}_{0.4} \mathrm{R} 0.649$ & 167.3 \\
\hline $\mathrm{P}^{+} \mathrm{F} \mathrm{MD}_{0.4} \mathrm{R} 0.727$ & 22.4 & 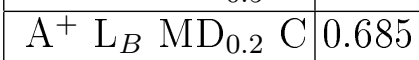 & 100.2 & $\mathrm{~A}^{+} \mathrm{F} \mathrm{ME_{0.5 } \mathrm { R }} 0.647$ & 158.5 \\
\hline 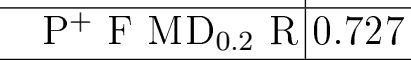 & 22.3 & 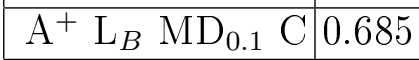 & 100.2 & $\mathrm{P}^{-} \mathrm{F} \mathrm{MJ} \mathrm{M}_{0.1} \mathrm{C} 0.643$ & 527.3 \\
\hline $\mathrm{W}^{+} \mathrm{F}_{B} \mathrm{MD}_{0.4} \mathrm{R} 0.726$ & 127.2 & \begin{tabular}{|l|l|l}
$\mathrm{W}^{+} \mathrm{F}_{B}$ & $\mathrm{MD}_{0.2} \mathrm{C}$ & 0.682 \\
\end{tabular} & 156.9 & $\mathrm{~A}^{+} \mathrm{F} \mathrm{MD}_{0.2} \mathrm{C} 0.643$ & 247.4 \\
\hline $\mathrm{P}^{+} \mathrm{F}_{B} \mathrm{MD}_{0.5} \mathrm{C} \quad 0.726$ & 40.1 & 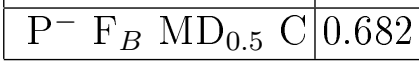 & 720.9 & 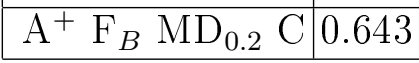 & 247.4 \\
\hline
\end{tabular}

Table S5: Pearson coefficients and $\chi^{2}$ values for all model variants, part 1 . 


\begin{tabular}{|c|c|c|c|c|c|c|}
\hline \begin{tabular}{l|l}
$\mathrm{D}_{0.5} \mathrm{R}$ & 0
\end{tabular} & 79. & $\mathrm{P}^{-} \mathrm{F}_{B} \mathrm{MJ}_{0.3} \mathrm{C} \mid 0$ & & $\mathrm{~W}^{+} \mathrm{F} \mathrm{MD}_{0.3} \mathrm{C}$ & & \\
\hline 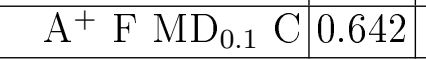 & 47. & 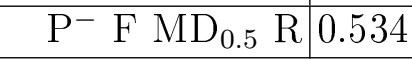 & 10 & $\mathrm{~W}^{-} \mathrm{F} \mathrm{MD}_{0.3} \mathrm{C}$ & & 43.2 \\
\hline 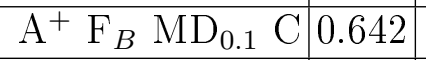 & 247. & 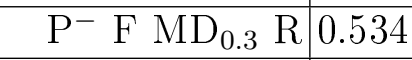 & 10 & $\mathrm{~W}^{+} \mathrm{F} \mathrm{MD}_{0.5} \mathrm{R}$ & 0 & 01 \\
\hline 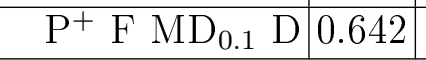 & 03.0 & $\begin{array}{ll}\mathrm{J}^{+} \mathrm{F}_{B} \mathrm{MD}_{0.5} \mathrm{R} & 0.531 \\
\end{array}$ & 933.3 & $W^{+} \mathrm{F} \mathrm{MD}_{0.1} \mathrm{R}$ & 0 & 01 \\
\hline $\mathrm{A}^{+} \mathrm{F} \mathrm{MD}_{0.4} \mathrm{C} \mid 0.642$ & 252.2 & $\begin{array}{ll}\mathrm{GC}, b=3.8 \AA & 0.531\end{array}$ & $>1000$ & $\mathrm{P}^{-} \mathrm{F} \mathrm{MJ}_{0.3} \mathrm{R}$ & 450 & $>1000$ \\
\hline \begin{tabular}{|l|l|}
$\mathrm{A}^{+} \mathrm{F}_{B} \mathrm{MD}_{0.4} \mathrm{C}$ & 0.642 \\
\end{tabular} & 252.2 & \begin{tabular}{ll|l}
$\mathrm{P}^{-} \mathrm{F}_{B}$ & $\mathrm{ME}_{0.3} \mathrm{R}$ & 0.527
\end{tabular} & 1000 & $\mathrm{~W}^{-} \mathrm{F} \mathrm{MD}_{0.5} \mathrm{R}$ & 148 & 638 \\
\hline $\begin{array}{l}\mathrm{P}^{+} \mathrm{F} \mathrm{MD} \\
0.1\end{array}$ & 682. & 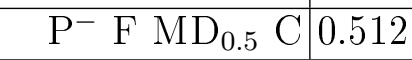 & 1000 & $\mathrm{~W}^{+} \mathrm{F} \mathrm{MD} \mathrm{MD}_{0.1} \mathrm{D}$ & 144 & 61. \\
\hline 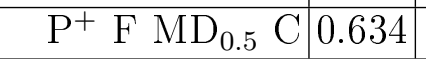 & 439. & 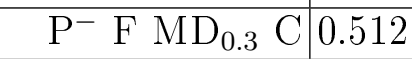 & 1000 & $\mathrm{P}^{-} \mathrm{F} \mathrm{MJ}_{0.3} \mathrm{C}$ & 44 & 1000 \\
\hline 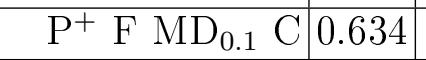 & 439.5 & \begin{tabular}{l|l|l}
$\mathrm{P}^{+} \mathrm{F} \mathrm{MD}_{1} \mathrm{R}$ & 0.511
\end{tabular} & $>1000$ & $\mathrm{~W}^{+} \mathrm{F} \mathrm{MD}_{0.5} \mathrm{C}$ & & 970 \\
\hline 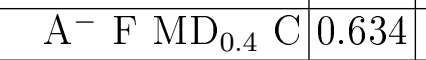 & 211.5 & 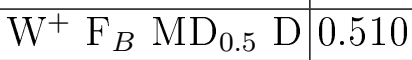 & 1000 & $\mathrm{~W}^{+} \mathrm{F} \mathrm{MD}_{0.1} \mathrm{C}$ & 43 & 970. \\
\hline 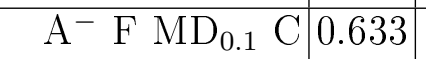 & 211.9 & $\mathrm{~V}^{+} \mathrm{F}_{B} \mathrm{MD}_{0.5} \mathrm{C} \mid 0.504$ & 10 & $\mathrm{P}^{+} \mathrm{F} \mathrm{MJ}_{0.3} \mathrm{R}$ & & 10 \\
\hline 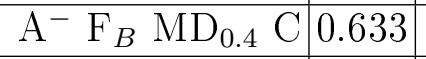 & 211.5 & 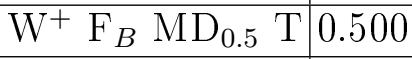 & 1000 & $\mathrm{~W}^{+} \mathrm{F} \mathrm{MD}_{0.1} \mathrm{~T}$ & 39 & 73.8 \\
\hline 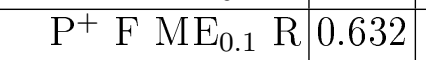 & 639.0 & 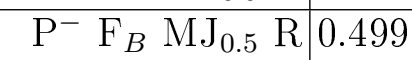 & 10 & $\mathrm{~W}^{-} \mathrm{F} \mathrm{MD}_{0.5} \mathrm{C}$ & & SO \\
\hline 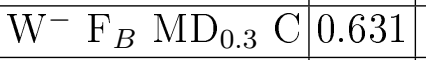 & 176.3 & 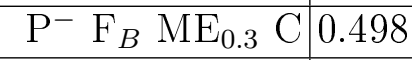 & $>1000$ & $\mathrm{P}^{+} \mathrm{F} \mathrm{MJ}_{0.3} \mathrm{D}$ & 35 & 1000 \\
\hline 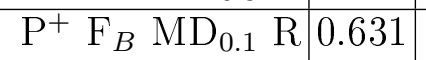 & $\overline{42.8}$ & \begin{tabular}{l|l}
$\mathrm{P}^{+} \mathrm{F} \mathrm{MD}_{1} \mathrm{D}$ & 0.497
\end{tabular} & 10 & $\mathrm{P}^{+} \mathrm{F} \mathrm{MJ}_{0.3} \mathrm{C}$ & & 0 \\
\hline 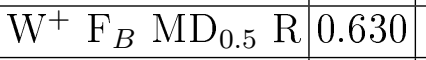 & 230.0 & 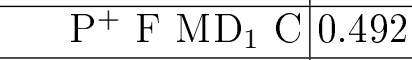 & $>1000$ & $\mathrm{P}^{+} \mathrm{F} \mathrm{MJ}_{0.3} \mathrm{~T}$ & 31 & $>1000$ \\
\hline 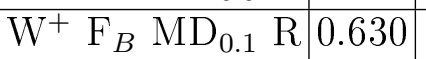 & 230.0 & 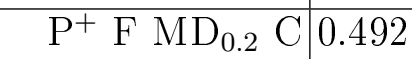 & $>1000$ & ${ }^{-} \mathrm{F}_{B} \mathrm{ME}_{0.5} \mathrm{R}$ & & \\
\hline 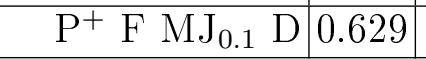 & 333.9 & $\begin{array}{l}\mathrm{W}^{+} \mathrm{F} \mathrm{MD} \\
\end{array}$ & 599.1 & $\mathrm{P}^{-} \mathrm{F} \mathrm{ME}_{0.3} \mathrm{R}$ & 21 & $>1000$ \\
\hline 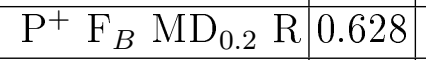 & 41. & \begin{tabular}{ll|l}
$\mathrm{P}^{+} \mathrm{F} \mathrm{MD}_{1} \mathrm{~T}$ & 0.486 \\
\end{tabular} & $>1000$ & $\mathrm{P}^{-} \mathrm{F}_{B} \mathrm{ME}_{0.5} \mathrm{C}$ & & $>1000$ \\
\hline \begin{tabular}{|l|l|}
$\mathrm{A}^{+} \mathrm{F} \mathrm{ME}_{1} \mathrm{~T}$ & 0.625 \\
\end{tabular} & 282.3 & \begin{tabular}{ll|l}
$\mathrm{P}^{+} \mathrm{L} \mathrm{ME}_{1} \mathrm{C}$ & 0.485 \\
\end{tabular} & 1000 & $\mathrm{P}^{-} \mathrm{F} \mathrm{ME}_{0.3} \mathrm{C}$ & 16 & $>1000$ \\
\hline $\mathrm{P}^{+} \mathrm{F} \mathrm{MJ}_{0.1} \mathrm{~T} \quad 0.623$ & 407.8 & $\begin{array}{l}\mathrm{W}^{+} \mathrm{F} \mathrm{MD} \\
0.2\end{array}$ & 548 & $\mathrm{~W}^{+} \mathrm{F} \mathrm{MD}_{0.5} \mathrm{R}$ & & $>1000$ \\
\hline 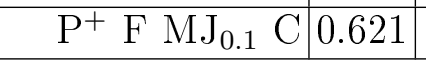 & 512. & \begin{tabular}{ll|l}
$\mathrm{W}^{-} \mathrm{F} \mathrm{MD}$ & $\mathrm{M} .1$ \\
$\mathrm{R}$ & 0.485 \\
\end{tabular} & 82 & $\mathrm{P}^{-} \mathrm{F} \mathrm{MJ}_{0.5} \mathrm{R}$ & & $>10$ \\
\hline 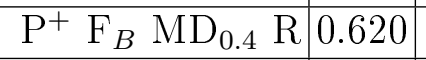 & 43.1 & $\begin{array}{l}\mathrm{W}^{-} \mathrm{F} \mathrm{MD} \\
0.4\end{array}$ & 432. & $\mathrm{P}^{+} \mathrm{F} \mathrm{ME}_{0.3} \mathrm{R}$ & & $>1000$ \\
\hline $\mathrm{P}^{-} \mathrm{F}_{B} \mathrm{MD}_{0.2} \mathrm{R} \mid 0$ & 43. & 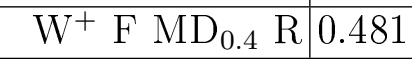 & & $\mathrm{P}^{+} \mathrm{F} \mathrm{ME}_{0.3} \mathrm{D}$ & & 100 \\
\hline 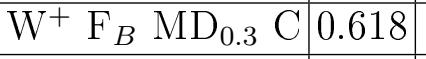 & 220.8 & 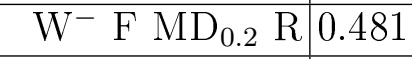 & 606.3 & $\mathrm{~W}^{+} \mathrm{F} \mathrm{MD}_{0.5} \mathrm{C}$ & 12 & $>1000$ \\
\hline $\mathrm{P}^{-} \mathrm{F}_{B} \mathrm{MD}_{0.1} \mathrm{R} \mid 0$ & 45. & 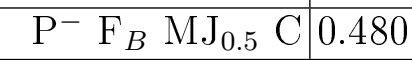 & 0 & $\mathrm{P}^{-} \mathrm{F} \mathrm{MJ}_{0.5} \mathrm{C}$ & & $>$ \\
\hline 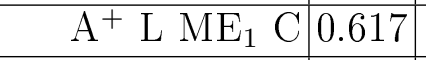 & 299.9 & $\begin{array}{l}\mathrm{W}^{+} \mathrm{F} \mathrm{MD} \\
\end{array}$ & 550.4 & $\mathrm{~W}^{+} \mathrm{F} \mathrm{MD}_{0.5} \mathrm{D}$ & 09 & $>1000$ \\
\hline $\mathrm{A}^{+} \mathrm{F} \mathrm{ME}_{0.5} \mathrm{C} \mid 0.612$ & 183.8 & 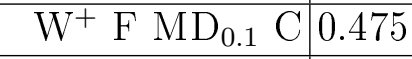 & 740 & $\mathrm{P}^{+} \mathrm{F} \mathrm{ME}_{0.3} \mathrm{C}$ & 08 & $>1000$ \\
\hline 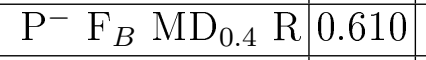 & 44. & 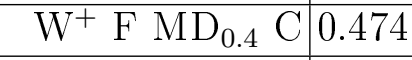 & 678. & $\mathrm{~W}^{+} \mathrm{F} \mathrm{MD}_{0.5} \mathrm{~T}$ & 08 & $>1000$ \\
\hline $\mathrm{P}^{-} \mathrm{F} \mathrm{ME}_{0.1} \mathrm{C}$ & 100 & 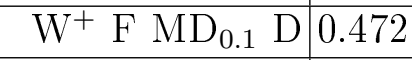 & 602. & $\mathrm{P}^{+} \mathrm{F} \mathrm{MJ}_{0.5} \mathrm{R}$ & 106 & $>1000$ \\
\hline \begin{tabular}{|l|l|}
$\mathrm{A}^{+} \mathrm{L} \mathrm{ME}_{1} \mathrm{~T}$ & 0.601 \\
\end{tabular} & 334 & $\begin{array}{l}\mathrm{W}^{+} \mathrm{F} \mathrm{MD} \\
\end{array}$ & 36 & $\mathrm{P}^{+} \mathrm{F} \mathrm{ME}_{0.3} \mathrm{~T}$ & 06 & $>1000$ \\
\hline $\begin{array}{l}\mathrm{P}^{+} \mathrm{F} \mathrm{ME} \\
\end{array}$ & 923.2 & 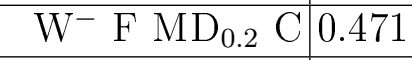 & 532 & $\mathrm{P}^{+} \mathrm{F} \mathrm{MJ}_{0.5} \mathrm{D}$ & 103 & $>1000$ \\
\hline 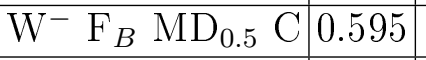 & 286.2 & 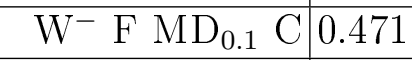 & 513 & $\mathrm{P}^{+} \mathrm{F} \mathrm{MJ}_{0.5} \mathrm{C}$ & 02 & $>1000$ \\
\hline 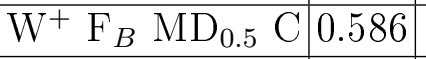 & 399.6 & 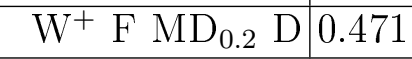 & 726 & $\mathrm{P}^{+} \mathrm{F} \mathrm{MJ}_{0.5} \mathrm{~T}$ & 0.401 & $>1000$ \\
\hline 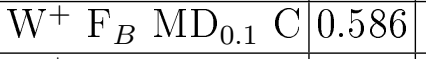 & 399. & 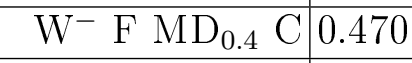 & 576 & $\mathrm{P}^{-} \mathrm{F} \mathrm{ME} \mathrm{ME}_{0.5} \mathrm{R}$ & 89 & $>1000$ \\
\hline 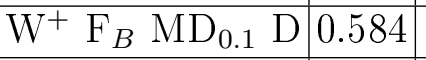 & 466. & 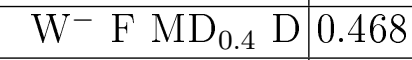 & 606 & $\mathrm{P}^{-} \mathrm{F} \mathrm{ME}_{0.5} \mathrm{C}$ & 86 & $>1000$ \\
\hline 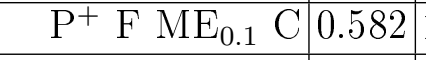 & $\$ 1000$ & 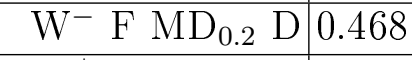 & 597. & $\mathrm{P}^{+} \mathrm{F} \mathrm{ME}_{0.5} \mathrm{D}$ & 0.384 & $>1000$ \\
\hline 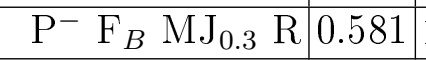 & $>1000$ & $\begin{array}{l}\mathrm{W}^{+} \mathrm{F} \mathrm{MD} \\
\end{array}$ & 770 & $\mathrm{P}^{+} \mathrm{F} \mathrm{ME}_{0.5} \mathrm{R}$ & 84 & $>1000$ \\
\hline 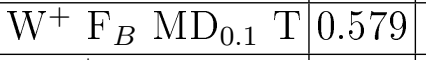 & 380.2 & 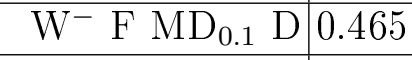 & 536. & $\mathrm{GC}, b=2.7 \AA$ & 0.382 & $>1000$ \\
\hline 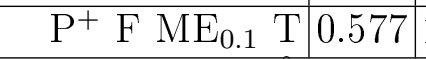 & $>100$ & 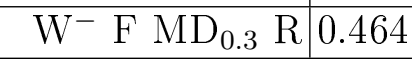 & 546. & $\mathrm{P}^{+} \mathrm{F} \mathrm{ME}_{0.5} \mathrm{C}$ & 51 & $>1000$ \\
\hline $\mathrm{GC}, b=4.1 \AA$ & 000 & 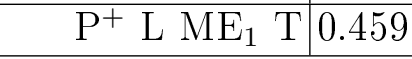 & 100 & $\mathrm{P}^{+} \mathrm{F} \mathrm{ME}_{0.5} \mathrm{~T}$ & 31 & $>1000$ \\
\hline
\end{tabular}

Table S5: Pearson coefficients and $\chi^{2}$ values for all model variants, part 2 . 


\subsection{SAXS profiles}

Fig. S12 shows a direct comparison of experimental SAXS data (magenta) with results of CG simulations of protein 6AAA (red). The best agreement is seen in the region of lowest scattering angles, with $q<0.15 \AA^{-1}$, which contains most of information on the overall shape and size of the protein.

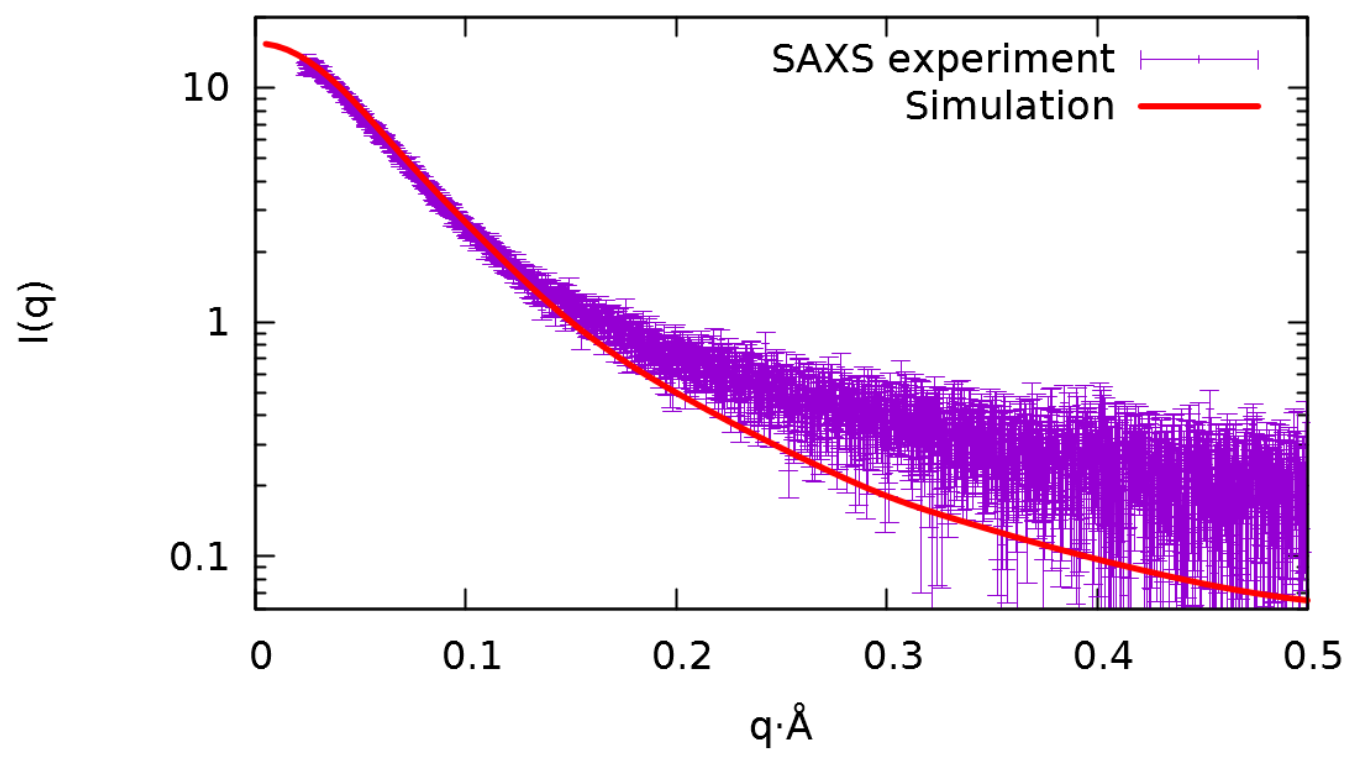

Figure S12: SAXS intensity $I$ as a function of momentum transfer $q$ measured experimentally for protein 6AAA [20] (magenta) and compared to simulation results (red). The simulation was carried out with the $\mathrm{P}^{-} \mathrm{F} \mathrm{MD}_{0.1} \mathrm{C}$ variant of the model. The scattering profile was computed for the 6AAA simulation trajectory using an algorithm co-developed with the EROS method [21]. Default parameters of the hydration shell on the protein surface were used. Intensity (in arbitrary units) was rescaled to match the experiment.

\section{Structured proteins}

We did not check how all 246 variants of our model perform for structured proteins, because the variants of the model best for structured proteins turned out to be very different from the top variants for IDPs - in the Table S6 we see that the smallest RMSD is achieved for interaction matrices multiplied by the factor 1 , whereas for IDPs this factor is smaller than 0.5 for the top models.

We measured Root Mean Square Deviation (RMSD) from the native structure of 3 proteins (1L2Y: 20 residues, 1ERY: 39 residues, 1UBQ: 76 residues) for simulations starting from a selfavoiding random walk or from the native state. In both cases the simulations lasted $150000 \tau$.

$1 \mathrm{~L} 2 \mathrm{Y}$

1ERY

1UBQ

\begin{tabular}{|c|c|c|c|c|c|c|c|c|}
\hline & & & & & & $\mathrm{Mc}$ & $\mathrm{SD}$ & \\
\hline $\mathrm{P}^{+} \mathrm{F}_{B} \mathrm{I}$ & $.0 \AA$ & & $\mathrm{P}^{-} \mathrm{F}_{B} \mathrm{ME}_{1} \mathrm{~T}$ & 7.0 & & $\mathrm{~T}$ & $1.1 \AA$ & \\
\hline $\mathrm{P}^{-} \mathrm{F}_{B} \mathrm{ME}_{1} \mathrm{~T}$ & $6.0 \AA$ & 6.0 & $\mathrm{~W}^{+} \mathrm{F}_{B} \mathrm{ME}_{1} \mathrm{~T}$ & $7.0 \AA$ & 6.3 & $\mathrm{P}^{+} \mathrm{F}_{B} \mathrm{ME}_{1} \mathrm{~T}$ & $11.3 \AA$ & $7 \AA$ \\
\hline $\mathrm{P}^{-} \mathrm{F} \mathrm{N}$ & $.1 \AA$ & .1 & $\mathrm{P}^{+} \mathrm{F}_{t}$ & $1.0 \mathrm{~A}$ & & & & \\
\hline${ }^{-} \mathrm{F} \mathrm{MD}_{0}$ & $6.2 \AA$ & 6.1 & $\mathrm{~W}^{+} \mathrm{L} \mathrm{ME}_{1} \mathrm{~T}$ & $7.0 \AA$ & 6.7 & $\mathrm{~T}^{-\mathrm{F} \mathrm{ME}} \mathrm{ME}_{1} \mathrm{~T}$ & 88 & $7.3 \AA$ \\
\hline $\mathrm{P}^{-} \mathrm{F} \mathrm{M}$ & $6.2 \AA$ & 6.1 & $-\mathrm{F} \mathrm{N}$ & $7.3 \AA$ & 6.7 & $F_{-1}$ & 11 & 3 \\
\hline $\mathrm{P}^{-} \mathrm{F}_{B} \mathrm{~N}$ & $6.2 \AA$ & 6.2 & $\mathrm{~W}^{-} \mathrm{F} \mathrm{MJ} \mathrm{MJ}_{1} \mathrm{~T}$ & $7.3 \AA$ & 6.8 & $\mathrm{P}^{-} \mathrm{F}_{B}$ & & .1 \\
\hline$\overline{\mathrm{P}^{-} \mathrm{F} \mathrm{I}}$ & $6.3 \AA$ & .3 & $\bar{F}$ & $7.5 \AA$ & 6 & $\mathrm{P}^{+} \mathrm{F}$ & $12.2 \AA$ & 9.3 \\
\hline $\mathrm{P}^{+} \mathrm{F}_{B} \mathrm{I}$ & $6.4 \AA$ & & $\overline{\mathrm{P}^{+} \mathrm{T}} \mathrm{M}$ & 7.5 & & $\mathrm{~W}^{-} \mathrm{L}$ & & \\
\hline $\mathrm{P}^{+} \mathrm{F} \mathrm{I}$ & $6.4 \AA$ & 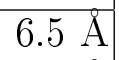 & & 7.6 & 6.1 & $-F$ & & 70 \\
\hline${ }^{-} \mathrm{L}_{B} \mathrm{MD}_{1} \mathrm{~T}$ & $8.6 \AA$ & $8.8 \AA$ & ${ }^{+} \mathrm{F}_{B} \mathrm{M}$ & $8.0 \mathrm{~A}$ & 7.4 & $\mathrm{P}^{-} \mathrm{L} \mathrm{ME}_{1} \mathrm{~T}$ & 12.8 & \\
\hline
\end{tabular}

Table S6: RMSD for 3 structured proteins (1L2Y, 1ERY and 1UBQ) for simulations starting from a self-avoiding walk (folding, $\left.\mathrm{F}_{\mathrm{RMSD}}\right)$ and from the native structure $\left(\mathrm{N}_{\mathrm{RMSD}}\right)$. 


\section{References}

[1] The function is made from two mirrored algebraic sigmoid functions, retrieved from: https://blob.pureandapplied.com.au/sigmoid-a-post-about-an-algebraic -function-im-having-too-much-fun/ (accessed January 15, 2020).

[2] Mioduszewski, Ł.; Cieplak, M. Disordered peptide chains in an $\alpha$-c-based coarse-grained model. Phys. Chem. Chem. Phys. 2018, 20, 19057-19070.

[3] Miyazawa, S.; Jernigan, R. L. Residue - Residue Potentials with a Favorable Contact Pair Term and an Unfavorable High Packing Density Term, for Simulation and Threading. J. Mol. Biol. 1996, $256(3), 623-644$.

[4] Betancourt, M. R.; Omovie, S. J. Pairwise energies for polypeptide coarse-grained models derived from atomic force fields. J. Chem. Phys. 2009, 130(19), 195103.

[5] Tozzini, V.; Trylska, J.; Chang, C. E.; McCammon, J. A. Flap opening dynamics in hiv-1 protease explored with a coarse-grained model. J. Struct. Biol. 2007, 157(3), 606-615.

[6] Holehouse, A. S.; Das, R. K.; Ahad, J. N.; Richardson M. O.G.; Pappu R. V. CIDER: Resources to Analyze Sequence-Ensemble Relationships of Intrinsically Disordered Proteins. Biophys. J. 2017,112(1), 16-21.

[7] Requião, R. D.; Fernandes, L.; de Souza, H.; Rossetto, S.; Domitrovic, T.; Palhano, F. L. Protein charge distribution in proteomes and its impact on translation. PLOS comp. biol. 2017, 13(5), e1005549.

[8] Kyte, J.; Doolittle, R. A simple method for displaying the hydropathic character of a protein. J. Mol. Biol. 1982, 157, 105-132.

[9] Cragnell, C.; Rieloff, E.; Skepö, M. Utilizing coarse-grained modelling and monte carlo simulations to evaluate the conformational ensemble of intrinsically disordered proteins and regions. J. Mol. Biol. 2018, 430(16), 2478-2492.

[10] Dignon, G.; Zheng, W.; Kim, Y.; Best, R.; Mittal, J. Sequence determinants of protein phase behavior from a coarse-grained model. PLOS Comp. Biol. 2018, 14(1), e1005941. [Table S2]

[11] Varadi, M.; Kosol, S.; Lebrun, P.; Valentini, E.; Blackledge, M.; Dunker, A. K.; Felli, I. C.; Forman-Kay, J. D.; Kriwacki, R. W.; Pierattelli, R.; Sussman, J.; Svergun, D. I.; Uversky, V. N.; Vendruscolo, M.; Wishart, D.; Wright, P. E.; Tompa, P. pE-DB: a database of structural ensembles of intrinsically disordered and of unfolded proteins. Nucl. Acids Res. 2014, 42(D1), D326-D335.

[12] Cragnell, C.; Durand, D.; Cabane, B.; Skepo, M. Coarse-grained modeling of the intrinsically disordered protein histatin 5 in solution: Monte carlo simulations in combination with SAXS. Proteins 2016, 84, 777-791.

[13] Rauscher, S.; Gapsys, V.; Gajda, M. J.; Zweckstetter, M.; De Groot, B. L.; Grubmüller, H. Structural ensembles of intrinsically disordered proteins depend strongly on force field: A comparison to experiment. J. Chem. Theory Comput. 2015, 11, 5513-5524.

[14] Mylonas, E.; Hascher, A.; Bernado, P.; Blackledge, M.; Mandelkow, E.; Svergun, D. I. Domain conformation of tau protein studied by solution small-angle x-ray scattering. Biochem. 2008, $47(39), 10345-10353$. 
[15] Kung, C. C.; Naik, M. T.; Wang, S.; Shih, H.; Chang, C.; Lin, L.; Chen, C.; Ma, C.; Chang, C.; Huang, T. Structural analysis of poly-sumo chain recognition by the rnf4-sims domain. Biochem. J. 2014, 462, 53-65.

[16] Chukhlieb, M.; Raasakka, A.; Ruskamo, S. Kursula, P. The N-terminal cytoplasmic domain of neuregulin 1 type III is intrinsically disordered. Amino Acids 2015, 47(8), 1567-1577.

[17] Moncoq, K.; Broutin, I.; Larue, V.; Perdereau, D.; Cailliau, K.; Browaeys-Poly, E.; Burnol, A.-F.; Ducruix, A. The pir domain of grb14 is an intrinsically unstructured protein: implication in insulin signaling. FEBS Lett. 2003, 554(3), 240-246.

[18] Wells, M.; Tidow, H.; Rutherford, T. J.; Markwick, P.; Jensen, M. R.; Mylonas, E.; Svergun, D. I.; Blackledge, M.; Fersht, A. R. Structure of tumor suppressor p53 and its intrinsically disordered n-terminal transactivation domain. Proc. Natl. Acad. Sci. USA 2008, 105, 240-246.

[19] Cordeiro, T. N.; Herranz-Trillo, F.; Urbanek, A. N.; Estaña, A. N.; Cortés, J.; Sibille, N.; Bernadó, P. N. Structural Characterization of Highly Flexible Proteins by Small-Angle Scattering. Adv. Exper. Med. Biol. 2017, 1009, 107-129.

[20] De Biasio, A.; Ibáñez de Opakua, A.; Cordeiro, T. N.; Villate, M.; Merino, N.; Sibille, N.; Lelli, M.; Diercks, T.; Bernadó, P.; Blanco, F. J. p15PAF is an intrinsically disordered protein with nonrandom structural preferences at sites of interaction with other proteins. Biophys. J. 2014, 106(4), 865-874. SAXS data was retrieved from https://web.archive.org/web/20160911130751/http://pedb. vib.be/accession.php? ID=PED6AAA (accessed May 12, 2020).

[21] Różycki, B.; Kim, Y. C.; Hummer, G. SAXS Ensemble Refinement of ESCRT-III CHMP3 Conformational Transitions. Structure 2011, 19(1), 109-116. 